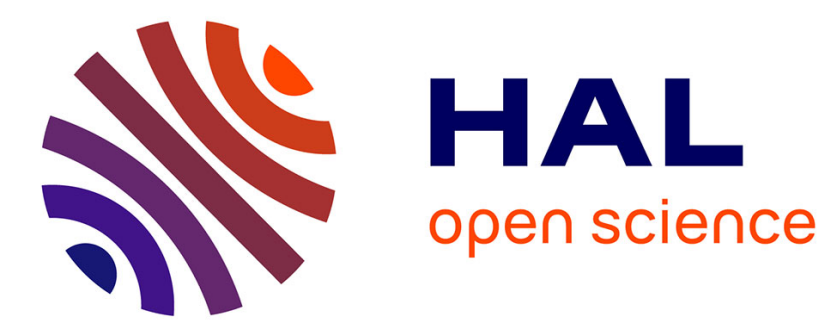

\title{
ISCCP Cloud Algorithm Intercomparison
}

\author{
W. Rossow, F. Mosher, E. Kinsella, A. Arking, M. Desbois, E. Harrison, P. \\ Minnis, E. Ruprecht, G. Seze, C. Simmer, et al.
}

\section{To cite this version:}

W. Rossow, F. Mosher, E. Kinsella, A. Arking, M. Desbois, et al.. ISCCP Cloud Algorithm Intercomparison. Journal of Climate and Applied Meteorology, 1985, 24 (9), pp.877-903. 10.1175/15200450(1985)0242.0.CO;2 . hal-03182913

\section{HAL Id: hal-03182913 \\ https://hal.science/hal-03182913}

Submitted on 27 Mar 2021

HAL is a multi-disciplinary open access archive for the deposit and dissemination of scientific research documents, whether they are published or not. The documents may come from teaching and research institutions in France or abroad, or from public or private research centers.
L'archive ouverte pluridisciplinaire HAL, est destinée au dépôt et à la diffusion de documents scientifiques de niveau recherche, publiés ou non, émanant des établissements d'enseignement et de recherche français ou étrangers, des laboratoires publics ou privés. 


\title{
ISCCP Cloud Algorithm Intercomparison
}

\author{
W. B. Rossow, ${ }^{*}$ F. MOSHER, ${ }^{* *}$ E. KINSElla,${ }^{\dagger}$ A. ARKING,${ }^{\S}$ M. Desbois,${ }^{\phi}$ E. HARRISON, ${ }^{a}$ \\ P. MinNIS, ${ }^{\alpha}$ E. RuPreCht,${ }^{* \phi}$ G. SEZE,${ }^{\ddagger}$ C. SIMMER, ${ }^{\|}$AND E. SMITH ${ }^{\prime}$ \\ *NASA Goddard Space Flight Center, Institute for Space Studies, New York, NY 10025 \\ ${ }^{* *}$ National Severe Storm Forecast Center, NOAA, Kansas City, MO 64106 \\ $\uparrow M A$ Com Sigma Data Inc., NASA/GISS, New York, NY 10025 \\ $\S N A S A$ Goddard Space Flight Center, Greenbelt, MD 20771 \\ - Laboratoire de Meteorologie Dynamique du CNRS, 91128 Palaisseau Cedex 05, France \\ ${ }^{\alpha} N A S A$ Langley Research Center, Hampton, VA 23665 \\ *\$ Institute fur Geophysik und Meteorologie, Universitat zu Koln, 5 Koln 41, Federal Republic of Germany \\ $\ddagger$ Laboratoire de Meteorologie Dynamique du CNRS, 91128 Palaisseau Cedex 05, France \\ "Los Alamos National Laboratory, Los Alamos, NM 87545 \\ = Department of Meteorology, Florida State University, Tallahassee, FL 32036
}

(Manuscript received 31 July 1984, in final form 5 April 1985)

\begin{abstract}
The International Satellite Cloud Climatology Project (ISCCP) will provide a uniform global climatology of satellite-measured radiances and derive an experimental climatology of cloud radiative properties from these radiances. A pilot study to intercompare cloud analysis algorithms was initiated in 1981 to define a state-ofthe-art algorithm for ISCCP. This study compared the results of applying six different algorithms to the same satellite radiance data. The results show that the performance of all current algorithms depends on how accurately the clear sky radiances are specified; much improvement in results is possible with better methods for obtaining these clear-sky radiances. A major difference between the algorithms is caused by their sensitivity to changes in the cloud size distribution and optical properties: all methods, which work well for some cloud types or climate regions, do poorly for other situations. Therefore, the ISCCP algorithm is composed of a series of steps, each of which is designed to detect some of the clouds present in the scene. This progressive analysis is used to retrieve an estimate of the clear sky radiances corresponding to each satellite image. Application of a bispectral threshold is then used as the last step to determine the cloud fraction. Cloudy radiances are interpreted in terms of a simplified model of cloud radiative effects to provide some measure of cloud radiative properties. Application of this experimental algorithm to produce a cloud climatology and field observation programs to validate the results will stimulate further research on cloud analysis techniques as part of ISCCP.
\end{abstract}

\section{Introduction}

Throughout the 1980s an international network of operational weather satellites will provide global radiance measurements in the visible $(0.6 \mu \mathrm{m})$ and thermal infrared $(11 \mu \mathrm{m})$ spectral intervals with a spatial resolution of at least $10 \mathrm{~km}$ and a time resolution of at least $3 \mathrm{~h}$. In July 1983 the International Satellite Cloud Climatology Project (ISCCP) began collection of a uniform global climatology of satellite measured radiances and will derive cloud properties to characterize the distribution and variation of clouds and their effects on Earth's radiation budget (Schiffer and Rossow, 1983). The climatological data obtained by ISCCP will be key elements in the study of cloud radiative feedbacks on climate and the improvement of climate model realism (GARP, 1975, 1978; Rossow, 1981). They will also prove useful for studies of other aspects of the climate involving clouds, such as the hydrologic cycle.

Selection and application of an operational cloud algorithm for ISCCP does not imply that the best method is already available, but is meant to stimulate research on improving methods of remote sensing and analysis of cloud properties. Thus, a major component of ISCCP is a research program to validate the operational cloud climatology and develop improved methods of analysis. This validation effort is part of a larger research program to investigate cloud-climate problems employing the radiance and cloud climatologies produced by ISCCP.

ISCCP research on cloud algorithms began with a pilot study initiated in late 1981 to evaluate currently available cloud analysis algorithms, to test the effects of data volume compression schemes, and to design the operational algorithm for ISCCP (World Climate Program, 1982b). A full resolution radiance data set from GOES-East and TIROS-N was sent to ten research groups and a program of analyses and tests was defined. Four Algorithm Intercomparison Workshops were held, the First in Ottawa, 31 May-1 June 1982 (World Climate Program, 1982a), the Second and Third in New York on 9-11 December 1982 and 7-8 April 1983, respectively (World Climate Program, 
1983a,b), and the Fourth in Greenbelt on 4-6 April 1984. Details of the data, analysis tasks and results of the Workshops and pilot study can be found in a report published in March 1984 by the World Meteorological Organization (World Climate Program, 1984).

This paper summarizes the pilot study by focusing on the most fundamental step of any cloud algorithm, that of cloud detection. Cloud detection is the process of deciding whether a particular radiance measurement represents a clear or cloudy scene. In the case of the algorithms considered here, cloud detection is evaluated by comparing the fractional cloud cover obtained by each method. Section 2 outlines the objectives and limitations of the pilot study and describes the criteria used to design the operational analysis algorithm. Section 3 describes the pilot study data set and the six algorithms most thoroughly evaluated. Section 4 presents the comparison of cloud cover fraction determinations, while Section 5 briefly discusses issues concerning the analysis of cloudy scenes to determine cloud radiative properties. Section 6 summarizes the conclusions of this study, describes the design of the ISCCP operational algorithm and presents recommendations for future studies.

\section{Objectives, limitations and design criteria}

Many different analysis algorithms have been suggested, all of which obtain useful information about clouds from satellite radiance data; but none of these methods have yet been used to produce a global climatology. Because the characteristics of the available satellite data preclude derivation of many cloud properties, and because current radiative transfer theory precludes calculation of some cloud effects on observations, and also because enough satellite data have not been examined to establish general patterns of cloud distribution and behavior, available analysis techniques must be evaluated to determine the best approach for obtaining the particular cloud properties (Table 1) that are the focus of ISCCP science objectives (World Climate Program, 1982b). In addition to this evaluation, the objectives of the pilot study included testing the performance of these methods when applied to radiance data which have been reduced in volume in various ways and determining the kind and magnitude of analysis errors associated with each compression technique.

Evaluation of the cloud algorithms is limited in two important ways. One objective of ISCCP is the production of a globally uniform climatology which resolves diurnal and seasonal cloud variations. Satellite radiometer design characteristics, together with practical limitations on manageable data volume, interact with the desires for global uniformity and sufficient time resolution to define data characteristics which limit analysis algorithm performance. Selection of the "best" analysis technique is thus constrained by the properties of the available data.
TABLE 1. Data specification for the International Satellite Cloud Climatology Project.

Parameters--Spatial and temporal averages and variances (or another statistical measure of the shape of the temporal distribution) are required for each of the following parameters.

Precision (30-day averages)

\section{Amounts}

Total cloud amount (fraction)*

Cirrus cloud amount (fraction)*

$\pm 0.03$

Middle cloud amount (fraction)

$\pm 0.05$

Low cloud amount (fraction)*

Deep convective cloud amount (fraction)

$\pm 0.05$

$\pm 0.05$

Height

Cirrus cloud-top height $(\mathrm{km})^{*}$

Middle level cloud-top height $(\mathrm{km})$

Low-level cloud-top height $(\mathrm{km})$

Deep convective cloud-top height $(\mathrm{km})$

Cloud-Top Temperature (K) for each

cloud category*

Cloud Optical Depth

Cloud Size Distribution

Average Narrow Band Radiances (VIS and IR)*

Spatial Averaging-The information is to be avèraged over approximately $250-\mathrm{km}$ by $250-\mathrm{km}$ boxes

Time Sampling-Every 3 hours, i.e., 8 times a day, centered around the synoptic observation times

Time Averaging-The global cloud climatology will consist of 30day averages for each of the 8 observing times per day

Length of Time Series- 5 years

* Highest priority

The second limitation on the evaluation is the lack of a "truth" data set against which to compare the results of each algorithm in order to judge performance. This problem is certainly not unusual in an experiment designed to make new observations, but it necessarily makes the evaluation of analysis methods much less direct or objective. Hence, the results presented in this paper are intercomparisons among the algorithms for many different cases to develop a general understanding of their relative performance. These intercomparisons are both statistical and specific in nature.

The pilot study did not lead to the selection of a particular algorithm for use by ISCCP, rather it led to definition of design criteria and to the design of a new algorithm based on elements of several methods. This occurred because the algorithms compared in this study were designed to accomplish somewhat different objectives and are in different stages of their development. Consequently, the pilot study intercomparisons were used to define the algorithm characteristics that "best" meet the ISCCP science objectives, given current understanding of the problem. Further research on analysis methods is expected to improve on the algorithm defined in this study (see Section 6f). The design criteria developed from the results, summarized in Section 4 and 5, are as follows: 
1) To construct a climatology of cloud radiative properties from available satellite radiance data necessitates utilization of both the visible and infrared wavelength measurements, when available (daytime), but the algorithm must work with only infrared data (nighttime). The requirement for monitoring diurnal cloud variations over the whole globe with the same type of data currently limits the data to two spectral channels near 0.6 and $11 \mu \mathrm{m}$ wavelengths.

2) To observe diurnal cloud variations over the whole globe and keep the total data volume manageable requires application of the algorithm to data which have degraded spatial distribution information. The algorithm must minimize sensitivity to this necessary data degradation.

3) To attain global uniformity in the climatology requires that the algorithm minimize cloud type and scene dependence in its results.

4) The behavior of the algorithm for simple and complex cases, for widely varying cloud types, and over all regions of Earth must be understood well enough to allow estimation of uncertainties.

In other words, the algorithm design described here represents compromises guided by the particular objectives of the project; other compromises are possible. In effect each set of choices made in designing an algorithm represents a distinct cloud model parameterizing actual cloud behavior; the particular parameterization adopted here is described in Section 6.

\section{Description of pilot test}

\section{a. Data set}

Collection of all of the raw imaging data from operational weather satellites would require nearly $200000(1600 \mathrm{bpi})$ data tapes per year for storage. This large volume is produced by high spatial resolution in visible channel images (from 1-4 km) and high time resolution (as high as every half hour). Data volume compression before analysis is clearly necessary. Since the lowest time resolution available among the current satellites is three hours, the first stage of the data compression is to reduce all satellites to three hour resolution, which has been found sufficient to describe average diurnal cloud variations (Harrison et al., 1983). The volume of all the data collected every three hours is still about 50000 tapes per year. Consequently, a second stage of the data compression is required that involves reduction of the spatial information. The pilot study data set was selected to allow study of different ways of reducing the spatial information and their effects on the cloud algorithm results.

The pilot study data set, distributed to all participants, includes both geosynchronous and polar orbiting satellite data. The GOES-East data are full resolution visible images $(0.9 \mathrm{~km}$ resolution at nadir, $0.5-0.7 \mu \mathrm{m}$ spectral interval) and infrared images $(6.9 \mathrm{~km}$ resolution at nadir, $10-12 \mu \mathrm{m}$ spectral interval) taken every three hours, starting with 0000 GMT, for 5-19 February 1979 . A visible channel radiance calibration is obtained from Norton et al. (1980). The infrared calibration is based on the NOAA NESDIS operational technique utilizing on-board reference targets. The TIROS- $\mathrm{N}$ radiance measurements $(4 \mathrm{~km}$ resolution at nadir, $0.55-0.9 \mu \mathrm{m}, 0.7-1.0 \mu \mathrm{m}, 3.6-4.0 \mu \mathrm{m}$ and $10.5-$ $11.5 \mu \mathrm{m}$ spectral intervals) once per day are for the same geographic regions. Calibration for these radiances are those operationally provided by NOAA NESDIS (Kidwell, 1981). The data cover three geographic regions, approximately $2000 \mathrm{~km}$ (or $20^{\circ}$ ) square centered on the coordinates: $40^{\circ} \mathrm{N}, 75^{\circ} \mathrm{W}$; (Region 1 , east coast of the United States and Canada); $2.5^{\circ} \mathrm{N}, 45^{\circ} \mathrm{W}$ (Region 2, northern coast of Brazil); and $30^{\circ} \mathrm{S}, 72.5^{\circ} \mathrm{W}$ (Region 3, west coast of Chile). Each region is subdivided into $2.5^{\circ}$ square boxes, 64 boxes covering the whole region (Fig. 1).

These regions and times were selected for study for two basic reasons. First, the three regions cover the basic climatological zones on Earth with both land and ocean areas represented: Region 1 represents winter midlatitudes with a continental east coast oceanic regime, Region 2 represents the tropical-subtropical regime including the ITCZ and trade wind regions, and Region 3 represents a subtropical-summer midlatitude region with a continental west coast oceanic regime. The most important exception to providing a complete climatological sample is the lack of a data set for a polar region; however, some relevant information is available in the analysis of the extreme northern part of Region 1. Second, the particular time period covered by the data was selected to include in Regions 1 and 3 some particularly challenging situations. Specifically, this time period includes a severe east coast snow storm followed by extremely cold, clear weather over Region 1 , producing a rapidly changing set of surface conditions with both large scale cloud systems and clear skies over the land. During the same period in Region 3, the cloudiness in the Andes varies from scattered thunderstorms to a fairly large storm system over Argentina. Consequently, the results presented in Section 4 represent the performance of these algorithms applied de-' liberately to some of the most difficult cloud types and weather situations.

\section{b. Cloud analysis algorithms}

All cloud algorithms consist of two basic steps: cloud detection and cloud analysis. The first step partitions the observed radiance values into those representing clear scenes and those representing cloudy scenes. Different algorithms are distinguished by the logic employed to make this choice. The second step involves the quantitative determination of cloud properties from the measured radiances. This step may be as simple as counting cloudy image pixels to obtain a single cloud parameter (e.g., fractional cloud cover) or as complex 

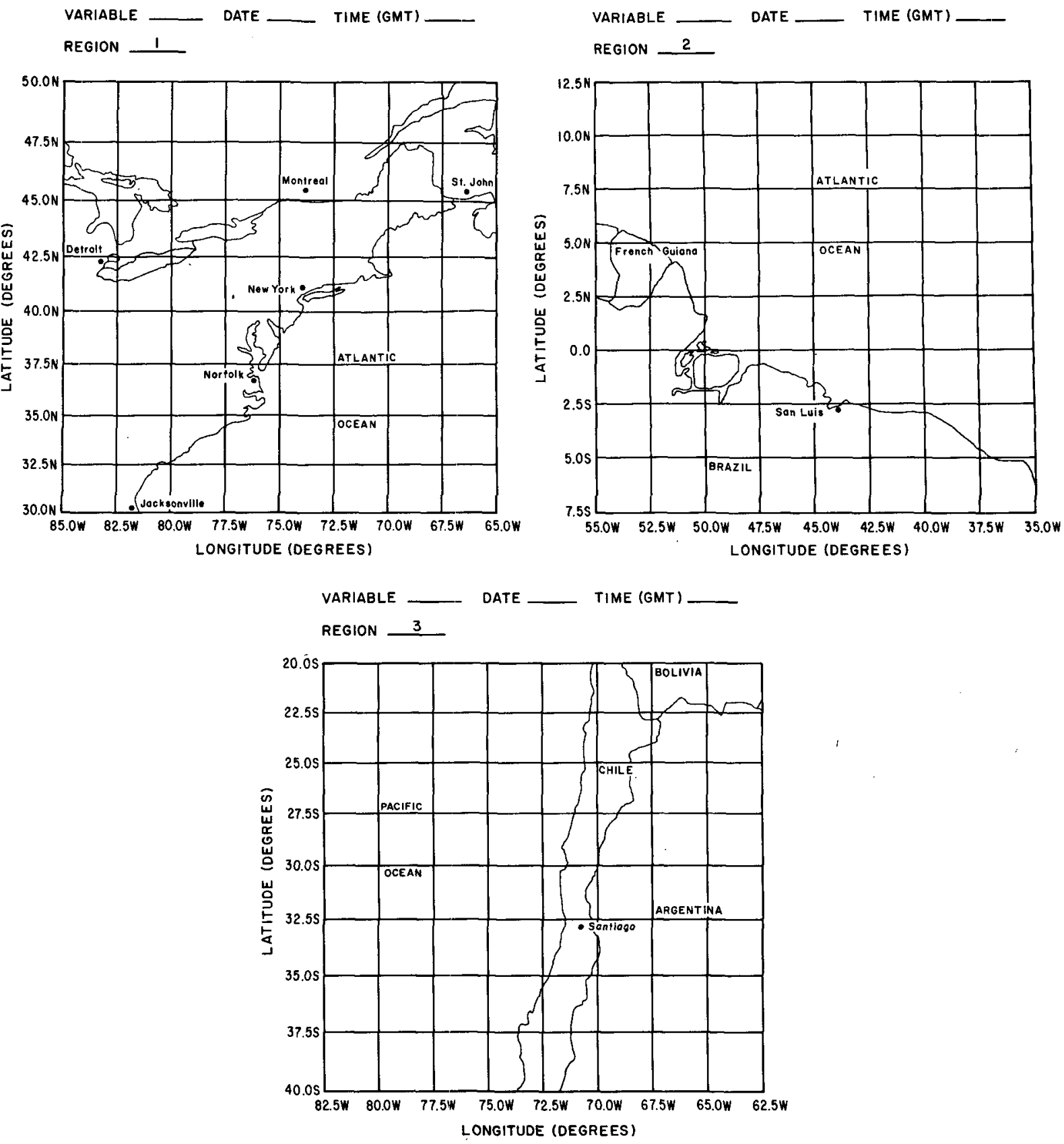

FIG. 1. Schematic maps of three pilot study test regions showing the standard $2.5^{\circ}$ square boxes.

as utilizing radiative transfer models to obtain a parameterized set of cloud properties. Underlying both of these basic steps is a radiative model; that is, each algorithm is built on assumptions regarding which atmospheric, surface and cloud properties affect the satellite measurements and by how much. In principle, although different algorithms may be distinguished by their respective radiative model, any radiative model can be employed to obtain cloud properties with any cloud detection logic. This is the reason for the focus on cloud detection methods in this paper.

A final distinction between algorithms is made according to whether the detection and analysis steps are performed on individual satellite image pixels or whether these steps are performed on pixel aggregations representing small subregions in the satellite image. The first type of algorithm is a threshold method; the second type will be referred to as a statistical method. 
The results presented here summarize detailed intercomparisons of the six methods applied to the largest amount of data (see World Climate Program, 1984). These particular algorithms are a visible threshold method (VIS TH), an infrared threshold method (IR TH), a bispectral threshold method incorporating a radiative transfer analysis (RT TH), a hybrid bispectral threshold method (HB TH), an asymmetric Gaussian histogram analysis (AG HIS) and a dynamic cluster histogram analysis (DC HIS). The first three of these methods are threshold techniques, whereas the last two are statistical methods. The fourth method is a hybrid approach, combining some features of threshold and statistical methods. All of these algorithms are being studied as part of research projects and do not represent the same stage of development.

\section{1) VISIBLE AND INFRARED THRESHOLD}

The simplest form of the threshold method classifies all image (visible or infrared) pixels as clear or cloudy according to whether the measured radiance $(R \text { or } T)^{1}$ differs from a clear sky value (RS or TS) by more than some predetermined threshold amount ( $\Delta R$ or $\Delta T)$. For visible (infrared) radiances a pixel is called cloudy if $R>\mathrm{RS}+\Delta R(T<\mathrm{TS}-\Delta T)$. The radiative model implied by this method assumes that the effects of the surface and atmosphere on the radiances are independent of time and viewing geometry to a precision determined by $\Delta R$ and $\Delta T$. The cloud cover fraction is then given by counting the number of cloudy pixels; that is, all cloudy pixels are assumed to be $100 \%$ cloud covered. The mean clear and cloudy radiances can then be taken to represent some cloud property; namely, $\mathrm{RC}=$ average $[R($ cloudy $)], \mathrm{RS}=$ average $[R($ clear $)]$, $\mathrm{TC}=$ average $[T($ cloudy) $]$ and TS $=$ average [ $T($ clear $)]$. More complicated cloud radiative models can be used to derive other quantities (e.g., albedo) from the cloudy radiance values.

The two methods presented here (VIS TH and IR TH) are more involved than suggested above because of the method used to select the clear sky radiances. The clear sky and cloudy radiances are assumed to form a monotonic distribution, with the clear sky as an extremum (minimum reflectivity or maximum brightness temperature). Fifteen-day radiance time records for each pixel location are examined to find the extreme values which are then taken to be representative of clear sky for the whole 15-day period. For a geosynchronous satellite, this approach can compensate for viewing geometry effects (which do not change in time) by obtaining a clear sky radiance for each location and time of day. A spatial filter is applied to the resulting clear sky radiance map to remove "noise

\footnotetext{
${ }^{1}$ Visible channel radiances are expressed as a reflectivity and IR channel radiances are expressed as brightness temperatures.
}

spikes" representing cloud contamination. For cloud detection, a threshold value representing about $3 \%$ reflectivity and $6 \mathrm{~K}$ in temperature is applied. Thus, for individual images, visible pixels more than $3 \%$ brighter than the clear sky or infrared pixels more than $6 \mathrm{~K}$ colder than the clear sky are counted as cloudy pixels. The resolution of the results from these threshold methods is limited only by the resolution of the satellite radiometer (or the data if they have been compressed by spatial averaging) and the frequency of the observations.

\section{2) RADIATIVE TRANSFER ANALYSIS}

This method (Rossow et al., 1985), though in effect a threshold method, differs in two respects from the above methods. First, for daytime data, this method applies both a visible and an infrared threshold, together. In this version of the method, both thresholds must be exceeded before a pixel is counted as cloudy. (At night, this method is similar to IR TH). Second, the clear sky radiance values are derived from a combination of statistical analysis of the time record of satellite observed radiances and conventional data sources. For the results presented here, RS over land is obtained from a statistical analysis of radiances from the NOAA-5 Scanning Radiometer and RS over water is based on a theoretical reflectivity model, while TS is obtained from the NMC global temperature and humidity analysis products.

In practice, this method compares the observed radiances to radiative transfer calculations using a model of the atmosphere, surface and clouds. The atmosphere is a Rayleigh scattering gas lying above and below a single cloud layer with a vertical temperature structure defined by the daily average NMC analyses. Radiation from the sun is partially absorbed by ozone distributed according to a zonal mean, seasonal climatology based on NIMBUS-4 BUV results (Hilsenrath and Schlesinger, 1981). Land surfaces are modeled as isotropic reflectors with reflectivities that vary with location. Snow cover brightening is included for Region 1 but based on data from a different year. Ocean reflectivity is calculated from Fresnel reflection coefficients with the wave-slope statistical model of Cox and Munk (1956). All surfaces are assumed to have unit emissivity in the infrared; but since surface temperatures are normalized to those measured by the satellite radiometer, this assumption has little effect. The single cloud layer is taken to be plane-parallel with reflectivity, transmissivity and emissivity characteristic of water spheres with an effective (weighted by cross section) mean radius of 10 $\mu \mathrm{m}$. Multiple scattering effects are included. The model radiances are, therefore calculated as a function of viewing geometry, cloud top pressure, surface reflectivity and temperature, vertical temperature and humidity distribution, ozone column abundance, and two cloud properties. 
Image pixel radiances are compared to the model radiances to obtain cloud optical thickness and cloud top temperature, assuming $100 \%$ cloud cover in each pixel. If cloud optical thickness is small, the corresponding infrared emissivity of the cloud is used to infer cloud top temperature. Cloud top altitude is also inferred using the vertical temperature profile from the NMC data. Unlike VIS TH or IR TH, where the threshold is a constant incremental radiance difference from the clear sky values, this method applies a threshold to the physical cloud properties obtained by comparison of the observed and model radiances. This means that the threshold values are not constant incremental radiances. If either cloud optical thickness or altitude is smaller than 0.4 or $0.5 \mathrm{~km}$, respectively, then no cloud is present.

\section{3) HYBRID BISPECTRAL THRESHOLD}

This method (Minnis and Harrison, 1984a,b,c) also applies both a visible and infrared threshold to daytime data (and becomes an IR TH method at night). It differs from RT TH in its rationale, in the way in which the thresholds are applied, and in the way that the radiative transfer analysis of cloudy pixels is performed. The HB TH was developed to estimate cloudiness over the entire diurnal cycle, so it relies more on the IR channel. However, it uses VIS radiances when available to tune the IR threshold to insure consistency between daytime and nighttime results (Minnis and Harrison, 1984a). The thresholds are applied to groups of pixels representing geographic subregions in the images; that is, the clear sky radiances, RS and TS, are assumed constant over the subregions. A map of RS is obtained from a minimum reflectivity analysis of the time record of the radiance measurements from GOES-East in November 1978. This map represents near noon observations and is assumed to be constant over the time period (usually one month). At other times of day RS is estimated from the near noon value with empirical bidirectional reflectance models developed from the time record analysis. These models depend on the surface type and solar zenith and satellite viewing angles. An average $\mathrm{RS}$ for each of the subregions is calculated; $\Delta R$ is about $1 \%$ reflectivity.

In each subregion, the average value of $T$ (call it $T^{\prime}$ ) for all pixels with $R<\mathrm{RS}+\Delta R$ is calculated. Pixels with small cloud amounts or thin cirrus can be included thus causing $T^{\prime}<\mathrm{TS}$. All pixels are summed in order of decreasing $T$ until their average value equals $T^{\prime \prime}$. The value of $T$ at which the summation ends is considered to be the threshold, TS $-\Delta T$. All pixels with $T<\mathrm{TS}$ $-\Delta T$ are counted as cloudy pixels, regardless of their $R$ values. Since low broken clouds can produce pixels with $T \geqslant$ TS $-\Delta T$, the value of TS $-\Delta T$ calculated in this manner will exclude some cloudy pixels. This procedure is assumed to offset, on average, the overestimate of CC (fractional cloud cover) caused by counting pixels as completely covered. This correction for the effect of partially filled fields-of-view is assumed correct, independent of cloud properties (see Minnis and Harrison, 1984a, for more discussion).

Before the thresholds are applied, all $R$ and $T$ values are normalized to remove angular dependence and atmospheric effects. Values of $R$ and RS are normalized by empirically determined bidirectional reflectance models for ocean, land and clouds with an overlying clear atmosphere. Values of $T$ are corrected by latitudinally and seasonally dependent limb darkening functions to remove water vapor absorption effects.

Once cloud fraction has been determined by counting cloudy pixels, clear pixels with $R<\mathrm{RS}+\Delta R$ and $T \geqslant$ TS $-\Delta T$ are used to calculate a surface temperature, assumed constant over the whole subregion. The value of RS is taken from the empirical models. The average cloud optical properties are then deduced from the values of CC, RS, TS, $R$ and $T$, all normalized as described and averaged over the subregion. A radiation balance that is linear in CC (Minnis and Harrison, 1984a) is employed to deduce RC and TC; i.e., $R=(1$ $-\mathrm{CC}) \mathrm{RS}+(\mathrm{CC}) \mathrm{RC}$ and $T^{4}=(1-\mathrm{CC}) \mathrm{TS}^{4}+(\mathrm{CC})$ $\mathrm{TC}^{4}$. These values represent a single effective cloud in each subregion. Wherever RC does not satisfy certain prespecified constraints, which are based on some percentage of the largest $R$ values or on an empirical model of $\mathrm{RC}$ depending on $\mathrm{CC}$, then $\mathrm{CC}$ is recalculated either with a VIS TH method using $\Delta R \approx 3 \%$ or with a specified value of $R C$ in the radiation balance equation. By defining cloud types in terms of temperature ranges tied to the value of TS by a constant lapse rate, these results can be obtained for low, middle and high cloud. The resolution of these results is established by the size of the subregion required for stable statistics of the image-pixels, usually about $250 \mathrm{~km}$ square.

\section{4) ASYMMETRIC GAUSSIAN HISTOGRAM ANALYSIS}

This statistical method (Simmer et al., 1982) forms a frequency histogram of radiance (6-bit count) values using a group of pixels from a geographic subregion in the images. No corrections for geometry or atmospheric effects are made. The two-dimensional histogram shows the frequency of occurrence of each correlated pair of visible and infrared radiances (at night the histogram becomes one-dimensional). The central analysis concept is that peaks in the one-dimensional histograms (visible and infrared) can be described by Gaussian distribution functions, the application of which identifies clusters in the $t w o$-dimensional distribution which represent homogeneous surface types (e.g., different cloud types or surfaces). The implicit radiative model is one in which different surfaces are sufficiently homogeneous over the subregion to form peaks in the histograms and different surfaces are represented by distinct radiance pairs.

Each pixel in the subregion is assigned to one of a 
number of two dimensional clusters which are defined by the following steps. (i) The maximum frequency peak in the IR histogram is identified. (ii) Two halfGaussian functions are fitted to the peak where the standard deviation on each side is derived by a regression between the radiances and the logarithm of the frequency. (iii) Pixels are assigned to this first cluster according to their distance from the peak radiance: namely, all pixels within one standard deviation, $\sigma$, are included in the cluster; all pixels more than $3 \sigma$ distant are excluded; a number of pixels equal to the extrapolated (Gaussian) frequency (or the total available, whichever is smaller) is included in the cluster for radiances between $\sigma$ and $3 \sigma$ distance from the peak radiance. (iv) Using only the pixels assigned to the IR cluster, a VIS histrogram is constructed and Gaussian functions fitted as before. (v) Step (iii) is applied to the VIS histogram, thus defining the first two-dimensional cluster. (vi) All pixels assigned to the first cluster are removed from the histogram. (vii) The steps i-vi are applied to the residual histogram to define a second cluster. If two clusters have overlapping one- $\sigma$ regions, they are combined into a single cluster. (viii) The procedure is repeated until all of the pixels are assigned to clusters or ten clusters are defined. (ix) If any pixels remain unassigned after ten clusters are defined, they are assigned to the nearest cluster defined by the shortest (two-dimensional) distance between the pixel and the cluster center.

Once all pixels are assigned to clusters and one cluster identified (if possible) as representing the actual surface, the fractional cloud cover, $\mathrm{CC}$, is given by the total number of pixels in all other clusters. In the results presented here, the identification of the actual surface is done by inspection, together with knowledge of the location of the subregion. The cloud and surface properties (RC, TC, RS, TS) are defined by the average radiances over the pixels within $\sigma$ of the peak frequencies of the appropriate cluster. The resolution of the results is given by the size of the subregion needed for stable statistics; the number of image pixels required is estimated to be $\sim 300$.

\section{5) DYNAMIC ClUSTER HISTOGRAM ANALYSIS}

This method (Desbois et al., 1982) also analyzes the two-dimensional (one-dimensional at night) frequency histogram of the radiances measured over a geographical subregion in the image; however, both the procedure for assigning pixels to clusters and the interpretation of the measured radiances are different. Unlike the previous method, dynamic cluster analysis isolates two-dimensional clusters defined by the population density in the histogram rather than clusters defined by peaks in two separate one-dimensional histograms. The central concept used to identify clusters is that every surface type in the subregion is represented in the histogram by a compact subdomain of the histo- gram; i.e., that all points in each subset are close to each other and that subdomains do not overlap. This is equivalent to the assumption that the radiance variation within each surface type or cloud is small compared with the radiance differences between types.

The clusters are identified by the following steps. (i) An initial set of clusters is chosen at random; this selection sets the maximum number [15] of clusters possible. (ii) For each initial cluster, a number of points [30] in the two-dimensional radiance space are selected at random to form the kernel of each cluster. (iii) The center of gravity (i.e., average radiance) and the standard deviation of the kernel point radiances are calculated. (iv) All other pixels in the histogram are assigned to the cluster with the nearest kernel (minimum distance between the pixel and the kernel center of gravity). If any cluster has too few pixels $[<50]$, it is eliminated. (v) The center of gravity and variance of each cluster are calculated using all the pixels in that cluster. (vi) This center of gravity and variance define a new kernel selection. (vii) Steps iii-vi are repeated until the differences between kernel and cluster centers of gravity stop changing.

All pixels are assigned to nonoverlapping clusters (see Desbois et al., 1982, for details). The fractional area covered by each surface type represented by a cluster is given by the number of pixels in that cluster. The actual surface is identified by comparing the radiance values at cluster centers of gravity to specified threshold values. In the results presented here, the surface clusters were determined by inspection of the whole data set. The fractional cloud cover $\mathrm{CC}$ is given by the total number of pixels in all clusters other than the surface clusters. The resolution of the results is given by the size of the subregion required for stable statistics; the number of pixels needed is estimated to be $>1000$.

The identification of histogram clusters was performed both on the $2.5^{\circ}$ boxes and on the larger $20^{\circ}$ area to compare the statistical effects. Using the larger area allows collection of a sufficient number of pixels for reliable statistics, even with degraded data, and enhances the probability that each cloud type or surface is represented by a sufficient number of pixels. The classification obtained on the larger area is then applied to the smaller subareas; this later approach was preferred in the pilot study results.

\section{Cloud detection intercomparison}

Since all of the methods considered here determine $\mathrm{CC}$ by counting cloudy pixels, intercomparison of CC values is a means of comparing the cloud detection ability of the algorithms. Four different methods of comparing $\mathrm{CC}$ distributions for each day and region were employed: differencing, regression, cross-correlation and manual inspection. The first three methods were used to compare results from each algorithm to results from VIS TH at $1 \mathrm{~km}$ resolution, to specific 
algorithm results, particularly $\mathrm{RT} \mathrm{TH}$, and to the average of all results. Space does not permit showing all of these comparisons; Fig. 2 and Table 2 provide a summary of the comparisons with specific illustrative cases discussed below.

Figure 2 shows a regression between individual $\mathrm{CC}$ values from each algorithm and the average of all methods; results are averaged over all three regions and over five days (see World Climate Program, 1984, for more details). Overall, the scatter of these averaged results varies about $\pm 7 \%$ around perfect correspondence (indicated by the solid line), with agreement generally better whenever CC is near zero or $100 \%$. This good agreement, despite methodological and developmental differences among the cloud algorithms, reflects the large number of cloudy situations where the clouds are sufficiently large scale compared with the data resolution and sufficiently distinct from clear sky that the methodological differences have only small effects on the results. This is confirmed by the spatial cross-correlations and biases between each individual result and the average of all results (Table 2). Overall the correlation coefficient for the spatial distribution is $\sim 0.9$ and the biases $<10 \%$ (even in an rms sense). Although comparison to the average of all results increases the correlation coefficient, the same conclusion is confirmed by correlations between particular algorithms which are still $\geqslant 0.7$ for most cases. Maps of the differences between $C C$ values in each individual case show most of the large differences at the edges of large scale cloud systems; i.e., disagreements are larger in partially cloudy scenes as Fig. 2 implies. Some of this "edge effect" appears to be due to differences in calculated latitude-longitude values between the groups; however, as discussed below, there are some methodological effects as well.

The conclusion illustrated in Fig. 2 and Table 2 must be emphasized: a substantial portion of the total cloud cover is properly detected by all algorithms tested because the clouds are large scale and high contrast in both the visible and infrared spectral channels. Nevertheless, some large disagreements do occur in these results, which are diagnostic of particular effects of methodology or of general difficulties in cloud detection. These problem cases, while representing a small fraction of the total, involve climatologically important

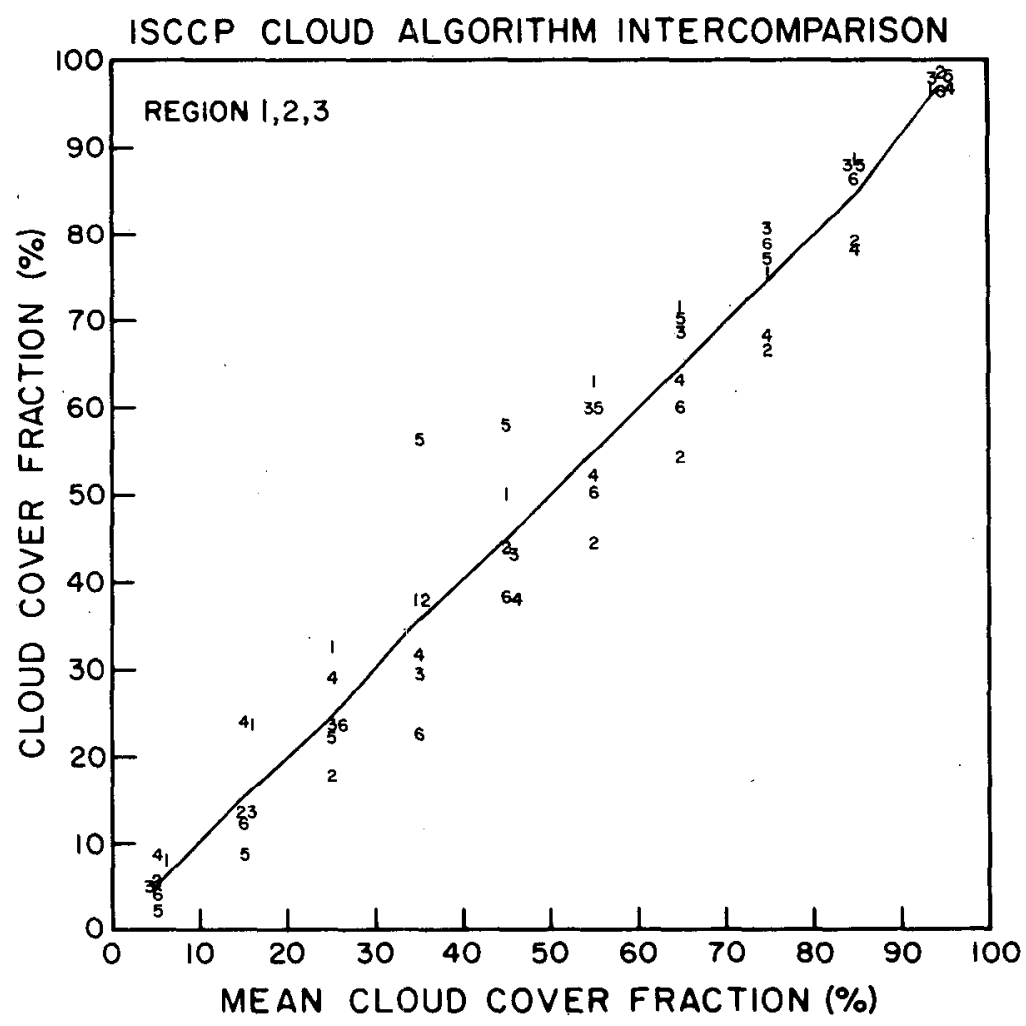

FIG. 2. Regression relation between cloud cover fraction obtained by each individual algorithm (ordinate) with the average of all methods (abscissa). The plotted values for each method represent the average value of cloud cover obtained by that method in all $2.5^{\circ}$ boxes with the cloud cover, averaged over all methods, equal to the value indicated on the abscissa. The methods are (1) VIS TH, (2) IR TH, (3) RT TH, (4) HB TH, (5) AG HIS and (6) DC HIS. Results averaged over all three regions for all five days (see text). 
TABLE 2. Correlation of cloud cover distribution over each study region obtained by each algorithm to the distribution averaged over all results. Numbers in the columns are normalized correlation coefficients with the bias in parentheses. Biases are in percent cloud cover.

\begin{tabular}{|c|c|c|c|c|c|c|}
\hline & VIS TH & IR TH & RT TH & HB TH & AG HIS & DC HIS \\
\hline \multicolumn{7}{|l|}{ Region 1} \\
\hline DOY 37 & $0.96(-3.5)$ & $0.99(+2.5)$ & $0.98(+3.1)$ & $0.93(-3.7)$ & $0.92(-1.7)$ & $0.93(+5.4)$ \\
\hline DOY 40 & $0.70(-0.6)$ & $0.91(-5.6)$ & $0.63(+8.5)$ & $0.89(-5.9)$ & $0.33(+18.2)$ & $0.88(-15.4)$ \\
\hline DOY 43 & $0.86(-1.7)$ & $0.83(+2.3)$ & $0.93(+0.4)$ & $0.90(-4.8)$ & $0.47(+16.2)$ & $0.84(-8.7)$ \\
\hline DOY 46 & $0.95(+7.2)$ & $0.60(-12.5)$ & $0.92(+1.2)$ & $0.51(-5.5)$ & $0.95(+1.2)$ & $0.96(+4.9)$ \\
\hline DOY 49 & $0.99(+3.4)$ & $0.99(-0.1)$ & $0.99(-2.5)$ & $0.92(+2.1)$ & $0.97(-0.9)$ & $0.98(-0.9)$ \\
\hline Average & $0.89(+1.0)$ & $0.86(-2.7)$ & $0.89(+2.1)$ & $0.83(-3.6)$ & $0.73(+6.6)$ & $0.92(-2.9)$ \\
\hline \multicolumn{7}{|l|}{ Region 2} \\
\hline DOY 37 & $0.92(+3.6)$ & $0.97(-2.4)$ & $0.98(+0.7)$ & $0.91(+10.4)$ & $0.96(-0.0)$ & $0.98(-6.2)$ \\
\hline DOY 40 & $0.79(+7.8)$ & $0.94(-7.5)$ & $0.94(+1.7)$ & $0.87(+7.3)$ & $0.87(-1.4)$ & $0.97(-2.7)$ \\
\hline DOY 43 & $0.91(+2.7)$ & $0.92(-11.4)$ & $0.96(+1.1)$ & $0.79(+4.0)$ & $0.90(+0.3)$ & $0.89(+3.1)$ \\
\hline DOY 46 & $0.80(+8.5)$ & & $0.91(-1.3)$ & $0.80(+7.8)$ & $0.68(+4.1)$ & $0.94(-8.7)$ \\
\hline DOY 49 & & & $0.89(+6.9)$ & $0.79(+9.6)$ & $0.67(+6.8)$ & $0.83(-15.3)$ \\
\hline Average & $0.86(+5.6)$ & $0.94(-7.1)$ & $0.94(+1.8)$ & $0.83(+7.8)$ & $0.82(+2.0)$ & $0.92(-6.0)$ \\
\hline \multicolumn{7}{|l|}{ Region 3} \\
\hline DOY 37 & $1.00(+0.9)$ & $0.84(-4.9)$ & $0.98(+7.9)$ & $0.97(-5.6)$ & $0.95(-5.2)$ & $0.99(+5.6)$ \\
\hline DOY 40 & $0.93(+0.1)$ & $0.86(-1.8)$ & $0.96(+1.6)$ & $0.97(-0.4)$ & $0.95(-3.9)$ & $0.90(+3.3)$ \\
\hline DOY 43 & $0.95(+1.8)$ & $0.85(-4.8)$ & $0.98(+1.7)$ & $0.97(-1.7)$ & $0.97(+0.6)$ & $0.98(+1.9)$ \\
\hline DOY 46 & $0.92(+2.3)$ & $0.87(-4.4)$ & $0.94(-0.5)$ & $0.95(-1.2)$ & $0.94(-1.8)$ & $0.97(+3.8)$ \\
\hline DOY 49 & $0.95(+2.4)$ & $0.43(-14.7)$ & $0.97(+3.3)$ & $0.90(+3.4)$ & $0.94(+2.5)$ & $0.98(+1.2)$ \\
\hline Average & $0.95(+1.5)$ & $0.77(-6.1)$ & $0.97(+2.8)$ & $0.95(-1.1)$ & $0.95(-1.6)$ & $0.96(+3.2)$ \\
\hline Total average & $0.90(+2.7)$ & $0.86(-5.3)$ & $0.93(+2.2)$ & $0.87(+1.0)$ & $0.83(+2.3)$ & $0.93(-1.9)$ \\
\hline
\end{tabular}

cloud types or situations. Thus, the focus of the discussion in this paper is on these more problematic cases in order to elucidate the workings of the algorithms and to suggest improvements for the ISCCP analysis.

Two classes of problems are discussed: 1) partially cloudy situations where cloudy radiances are not very different from clear sky radiances in one or both of the radiometer spectral channels, and 2) situations where complex variations in the clear sky (surface) properties make their accurate determination difficult. Both of these situations can be considered to be "low contrast" in that the detection signal (the difference between the measured and clear sky radiances) is small compared to the uncertainties in these radiances. Consideration of specific cases in all three regions can be used to separate the effects of the method used to determine clear sky radiances from the effects of the process used to make the cloudy-clear decision.

Figures 3, 4 and 5 display the results of Fig. 2 for pairs of algorithms and also show results by region. In each figure, the results from the two algorithms are displayed as broken lines, while the average over all six methods is shown as a solid line. Figure 3 shows results from VIS TH and IR TH, Fig. 4 shows HB TH and RT TH, and Fig. 5 shows DC HIS and AG HIS. These figures suggest some methodological differences that are further examined below.

The IR TH cloud amount is generally less than the VIS TH value (Fig. 3a), primarily because IR TH misses low-lying clouds over the ocean in Regions 2 and 3 (Figs. 3c and 3d). In Region 1, IR TH obtains larger values of $\mathrm{CC}$ because the clear sky radiance used for all five days is the maximum IR radiance observed at each location during the 15-day record. Since a strong storm passed over the region followed by clear, very cold weather, the IR TH method mistakes these cold surface temperatures for cloud. On the other hand, the snow cover produced by this storm causes an overestimate of CC by VIS TH because the 15-day minimum reflectivity is used as the clear sky value for all five days. Overall the largest disagreements between the methods occur over cold, snow-covered land in Region 1 and over oceans in Region 2, where the very lowlevel clouds are both warm and relatively dark.

Figure 4 shows that, in general, the two bispectral threshold methods agree better than the two single channel methods, probably resulting from the compromises between the VIS and IR threshold decisions. However, Figs. $4 \mathrm{c}$ and $4 \mathrm{~d}$ show that there are some differences between these methods which can be attributed to differences in the precise way that the thresholds are applied. The RT TH method requires that the measured radiances exceed both the VIS and IR thresholds for an image pixel to be classified as cloudy, whereas the HB TH utilizes the IR radiances of all image pixels below a VIS threshold to set an IR threshold. As discussed later, the warm, relatively dark clouds in Region 2 are generally missed by the RT TH 
method and may be overestimated by the HB TH method, while the slightly colder and brighter clouds in Region 3 (Fig. 4d) produce much better agreement between the methods. This different treatment of lowlying clouds by the two methods is the main reason for the tendency of the CC values of RT TH to be higher than those of HB TH.

Figure 5 compares the results from the two statistical methods, which are similar in their use of clusters in the two-dimensional radiance histogram to identify clouds, but are different in their methods of cluster definition. For the results shown here, the two methods also differ in the spatial scale on which the cluster classification is performed: DC HIS results are from classification of the whole region while AG HIS results are from classifications of individual $2.5^{\circ}$ box. These two methods compare about as well as the two bispectral threshold methods except for Region 1 . The disagreement in Region 1 shows no consistent pattern as a function of cloud-clear sky radiance contrast (see World Climate Program, 1984). As illustrated by specific cases, the disagreements of these two methods are scene dependent, growing large when the cloud--clear sky radiances form complex or ambiguous histograms. This is particularly true for partial cloud cover over snow-covered land.
Discussion of specific cases (Table 3 ) is divided by the type of surface forming the background in the satellite image data.

\section{a. Homogeneous background}

The ocean is a nearly homogeneous surface on spatial scales $\sim 100 \mathrm{~km}$ which does not change rapidly in time. Consequently, the clear sky radiances over ocean regions are nearly constant over the whole scene (one $2.5^{\circ}$ square subregion) and from one day to the next as long as the viewing geometry is nearly constant. Examination of these simple cases in the pilot data set decreases the importance of the accuracy of the clear sky radiance specification, RS and. TS, and isolates methodological differences between algorithms. For all the specific cases discussed in this section, the $1 \mathrm{~km}$ VIS TH results have been adjusted by the algorithm working group by visual inspection of VIS and IR photographs with different exposures. These VIS TH results represent the best available estimate of the actual cloud amount in these regions. The VIS TH results included in Figs. 2 and 3 are for $8 \mathrm{~km}$ resolution data and differ by small amounts from the values shown in Table 3 for the specific cases.
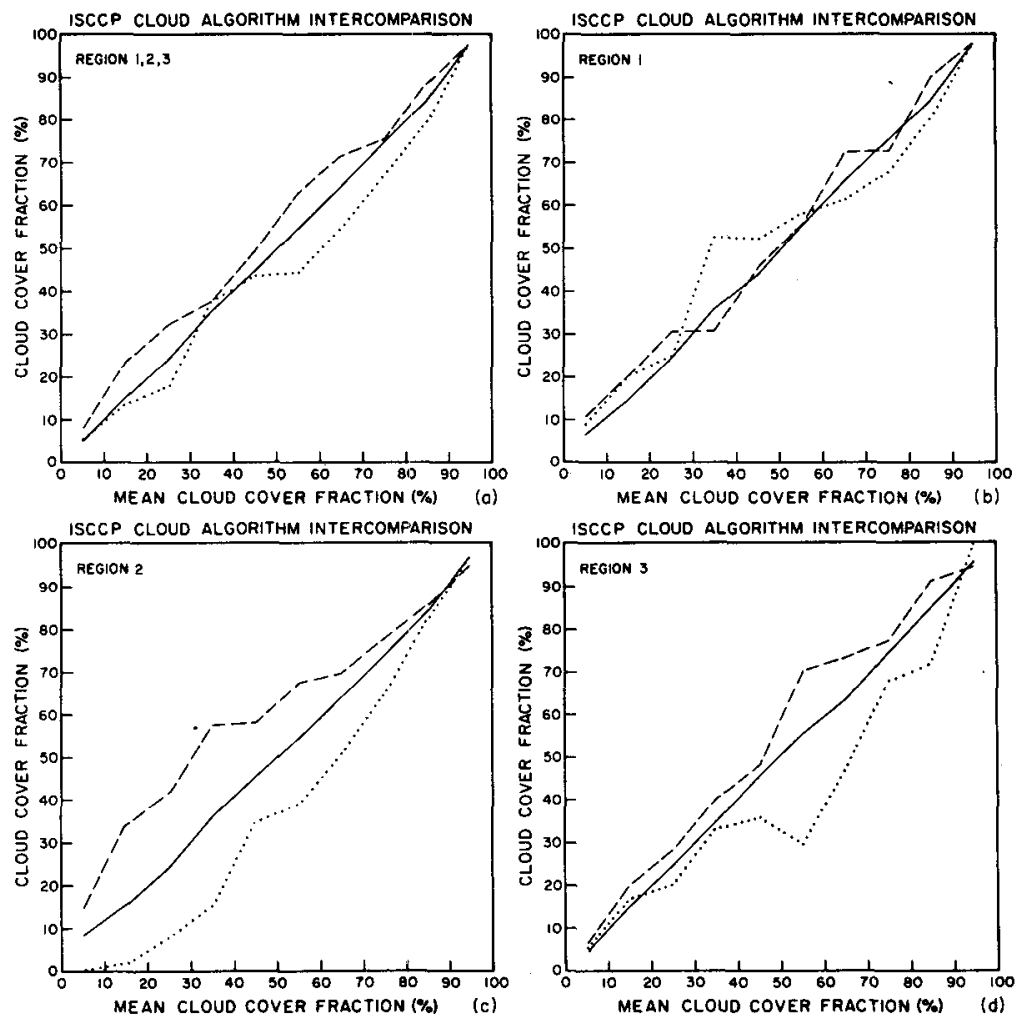

FIG. 3. Five-day average cloud cover fraction obtained by the VIS TH (dashed line) and IR TH (dotted) methods cornpared to the average of all methods (solid line) for (a) all three regions, (b) region 1, (c) region 2 and (d) region 3. 

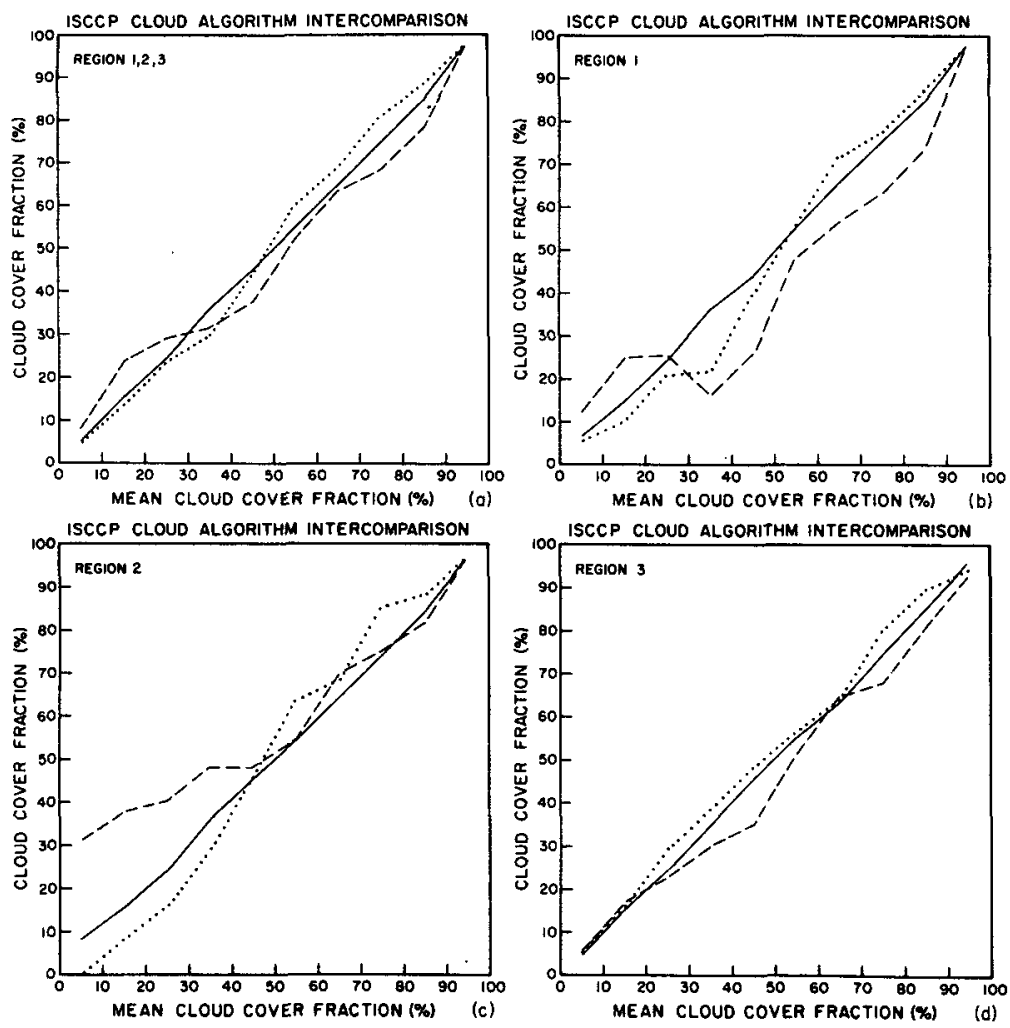

FIG. 4. As in Fig. 3 but showing only results from the HB TH (dashed) and RT TH (dotted) methods.
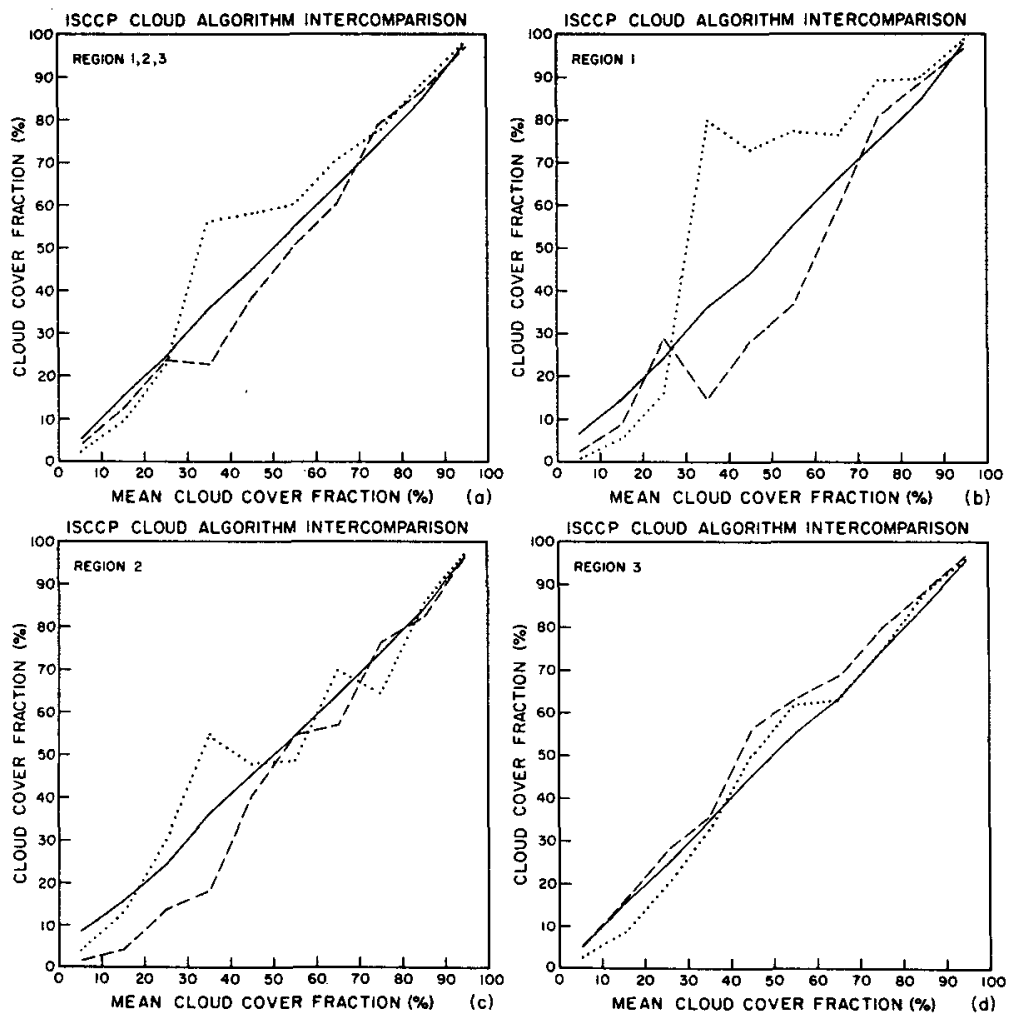

FIG. 5. As in Fig. 3 but showing only results from the DC HIS (dashed) and AG HIS (dotted) methods. 
TABLE 3. Comparison of cloud cover amounts retrieved by the different algorithms for $19\left(2.5^{\circ}\right)$ subregions.

\begin{tabular}{|c|c|c|c|c|c|c|}
\hline $\begin{array}{c}\text { Box } \\
\text { number }\end{array}$ & $\begin{array}{c}\text { VIS TH } \\
(1 \mathrm{~km})\end{array}$ & IR TH & RT TH & HB TH & AG HIS & DC HIS \\
\hline $1-37-1 / 2$ & 42 & 90 & 21 & 35 & $58(51)$ & $48(51)$ \\
\hline $1-37-1 / 3$ & 14 & 62 & 12 & 16 & $22(38)$ & $15(18)$ \\
\hline $1-37-3 / 2$ & 39 & 94 & 85 & 69 & $96(79)$ & $100(100)$ \\
\hline $1-37-4 / 5$ & 7 & 21 & 15 & 7 & $5(11)$ & 7 (13) \\
\hline $1-37-5 / 5$ & 65 & 81 & 84 & 87 & $89(78)$ & 81 \\
\hline $1-37-8 / 6$ & 72 & 72 & 67 & 77 & $84(83)$ & $58(79)$ \\
\hline $1-37-8 / 7$ & 65 & 58 & 39 & 60 & $98(92)$ & $26(27)$ \\
\hline $2-37-2 / 4$ & & & 0 & & & . (10) \\
\hline $2-37-3 / 6$ & 15 & 0 & 1 & 32 & $2(10)$ & $0(8)$ \\
\hline $2-37-4 / 6$ & 62 & 34 & 47 & 63 & $57(62)$ & $36(66)$ \\
\hline $2-37-6 / 2$ & 99 & 100 & 100 & 100 & $100(100)$ & $100(100)$ \\
\hline $3-37-2 / 4$ & 25 & 14 & 33 & 24 & $25(25)$ & $30(30)$ \\
\hline $3-37-3 / 4$ & 32 & 8 & 59 & 25 & $21(25)$ & $49^{\prime}$ \\
\hline $3-37-4 / 4$ & 61 & 27 & 66 & 41 & $46(43)$ & $61(67)$ \\
\hline $3-37-4 / 7$ & & 38 & 35 & 22 & $24(27)$ & $34(32)$ \\
\hline $3-37-5 / 4$ & 55 & 25 & 71 & 47 & $57(50)$ & $68(68)$ \\
\hline $3-37-6 / 3$ & 70 & & 45 & 44 & $35(36)$ & $60(59)$ \\
\hline $3-37-6 / 4$ & 32 & & 24 & 30 & $20(20)$ & $30(30)$ \\
\hline $3-37-7 / 4$ & 0 & 15 & 0 & 0 & $0(0)$ & $16(17)$ \\
\hline
\end{tabular}

Most of the southern portion of Region 1 on 6 February 1979 (day of year 37 ) is covered by large-scale stratus clouds associated with a developing storm system moving rapidly up the east coast of the United States. Figure 6 isolates a test case over the ocean which is on the edge of this large-scale cloud. The clouds to the west have tops at middle levels $(3-5 \mathrm{~km})$ but the broken cloud to the east lies at altitudes $<3 \mathrm{~km}$. The separate cluster in the histogram in Fig. 6 shows that the cloudy radiances are generally distinct from the clear-sky radiances, but the low broken clouds produce a broad distribution of pixels between the surface and cloud clusters. In Table 3 , all of the threshold methods agree with the VIS TH results reasonably well, but the statistical methods disagree both with VIS TH and with each other. The two different methods of assigning the scattered pixels to clusters seem to be responsible for this behavior of the statistical methods.

The region just to the east has only the low-lying clouds (Fig. 7), thus, many more pixels are near the clear-sky cluster, making it more difficult for all the methods in this case to decide between completely cloudy or completely clear sky. The cluster boundaries determined by the two statistical methods include different pixels in the clear-sky cluster and lead to the large disagreement. On the other hand, the threshold methods which rely more on the infrared threshold miss more of the broken clouds in the second case compared to the first. In Fig. 7 the difficulty of choosing the correct IR threshold can be visualized by trying to draw a vertical line through the histogram that correctly separates clouds from surface. The HB TH method tries to compensate for this IR threshold difficulty by allowing any pixels in the lower right quadrant (corresponding to cold, dark clouds) to influence the location of the vertical line representing the IR threshold.
Table 3 indicates that this approach produced a more accurate result for the case in Fig. 7.

Figure 8 illustrates a more extreme (and difficult) case of low broken cloud. This area in Region 2 is north of the winter ITCZ extending along the equator from the mouth of the Amazon river eastward over the tropical Atlantic and is generally covered by very low altitude, trade-wind cumulus clouds with very low reflectivities. Figure 8 shows that these clouds, capping a very moist boundary layer, are indistinguishable (at $2 \mathrm{~K}$ resolution in brightness temperature) from "clear sky" in the infrared. Indeed, this boundary is so hazy that it is difficult even in the visible to pick out clouds; the histogram in Fig. 8 shows the low reflectivity of these clouds. The results in Table 3 show that the threshold methods relying primarily on the IR do not detect these clouds. (Wielicki and Coakley, 1981, illustrate the same general problem). The statistical methods also do not detect a separate cloud cluster in this extreme case. However, use of a VIS threshold successfully detects the presence of some clouds, but the disagreement between VIS TH and HB TH depends on precisely how "dark" a reflectivity is considered to represent cloudy conditions.

Figure 9 shows a different type of low cloud in Region 3 over the Pacific off the coast of Chile. The stratocumulus clouds in this region are not only composed of larger individual clouds, but also of brighter (thicker) clouds in the visible. The comparison of the algorithm results for this case and that shown in Fig. 8 illustrates the role of the magnitude of the threshold radiance in the algorithm results. Whereas the IR threshold employed by most of the methods in the Fig. 8 case caused most methods to miss the clouds completely, in Fig. 9 the smaller IR threshold for RT TH produces higher cloud fractions than IR TH. On the other hand, the 

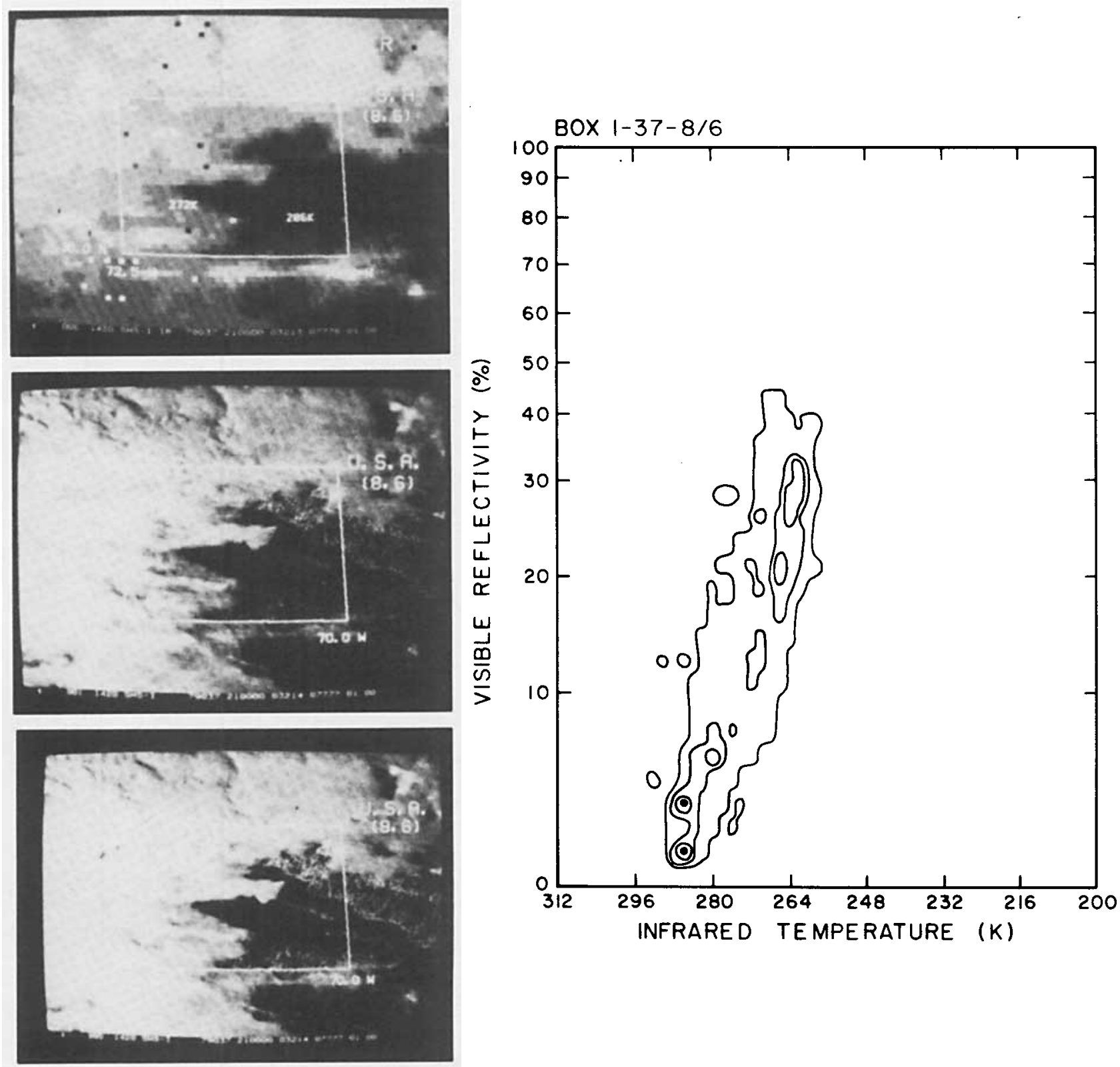

FIG. 6. Data for subregion 1-37-8/6 (see text for numbering convention): (upper photo) IR radiance data showing particular box, (middle photo) VIS image data, (lower photo) VIS image showing only data above VIS threshold. Two-dimensional radiance histogram shows frequency of occurrence of VIS radiances expressed as percent reflectivity (vertical axis) and IR radiances as brightness temperature (horizontal axis). Contours show equal increments in frequency. Results of applying different algorithms shown in Table 3.

method HB TH uses to select its IR threshold produces an apparent overestimate of cloud fraction in the former case, while producing an underestimate in the latter case.

The histograms in Figs. 9 and 10, exhibit small differences in the detailed frequency distribution of the radiances even though the general histogram shapes are similar. These subtle differences, attributable to small changes in cloud properties such as size distribution, lead to changed relationships among the al- gorithm results. Whereas the RT TH and DC HIS results overestimate cloud fraction (relative to VIS TH) in Fig. 9, these methods underestimate the cloud amount in Fig. 10. All of the methods failed to detect properly a $15 \%$ increase in cloud fraction between the two scenes.

Taken together, all of the cases discussed above highlight the interaction between cloud properties, especially for broken clouds, and the methodology used to separate clear and cloudy pixels; that is, all of the 


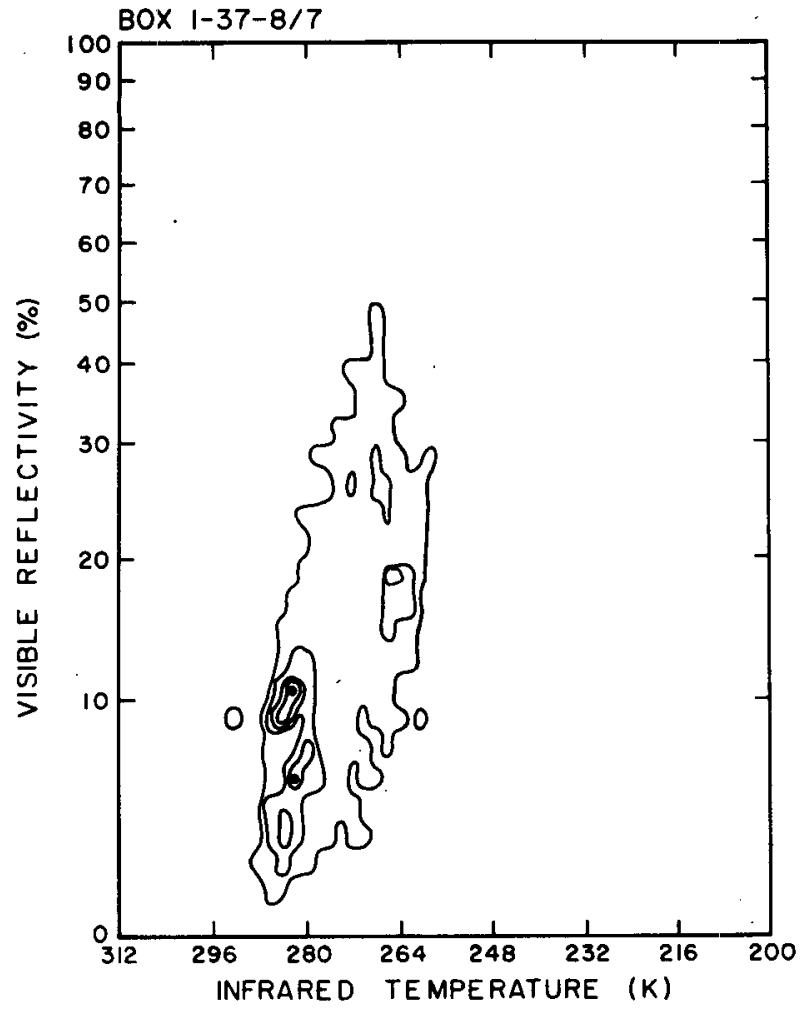

FIG. 7. Radiance histogram for subregion $1-37-8 / 7$ (see caption to Fig. 6)

methods exhibit some scene/cloud type dependence. This interaction occurs because satellite radiometer fields-of-view (FOVs) are generally larger than individual cloud elements in broken cloud fields, so that varying coverage of the FOVs produces a wide scatter of radiance values. (Partial coverage is not the only cause of scatter, however, as discussed in Section 5.) In such circumstances, the differences among the methods lie in their ability to separate completely clear pixels from partially cloudy pixels. For the threshold methods this ability depends both on the magnitude of the threshold radiance (assuming that the RS and TS are accurately known) and on the logic used to apply the thresholds as the cases discussed above illustrate. For the statistical methods this ability depends on the cluster definition procedure.

Judged from the standpoint of cloud detection alone, the threshold methods seem better because, in principle, use of small thresholds allows detection of small radiance variations away from clear sky values (which must be known accurately), whereas current cluster identification techniques cannot separate closely spaced radiance values. The threshold logic can also be designed to allow detection of "single channel" clouds, i.e., those clouds detectable only in a single spectral channel. Low-lying clouds are generally more easily detected in the VIS channel, as illustrated above (see also, Chahine, 1982; Saunders, 1985), whereas cirrus (not shown) is often easier to distinguish in IR. Neither RT TH nor HB TH handled either of these cases properly, however.

The above remarks cannot be interpreted as inherent limitations on any of these methods, however, because they have all been designed to work even though RS and TS are not well-known, and to overcome the effects of low spatial resolution. The latter factor means that counting every pixel in which some cloud is detected as $100 \%$ covered will overestimate the total cloud cover in a region (see also, Coakley and Bretherton, 1982). The statistical methods and HB TH try to compensate for this effect by grouping some cloudy pixels with the clear sky pixels; that is, those pixels with radiance values "close" to the clear sky values are presumed to be mostly clear, while those pixels with radiance values "close" to the "completely cloudy sky" values are presumed to be completely cloudy. Threshold methods also attempt this same trade of mostly clear for mostly cloudy pixels by using larger values of $\Delta R$ and $\Delta T$. The CC values shown in Table 2 in parentheses for the two statistical methods represent the changes produced by adjustments in the decision criteria (AG HIS) or by application of the cluster analysis to a different scale (DC HIS), illustrating the dependence of results on these decision criteria. Tests of VIS TH results show changes of $\mathrm{CC} \sim 10 \%$ for changes in the threshold of $\sim 3 \%$. The key conclusion is that the methodological and scene dependent behavior illustrated by the cases above arises from different algorithmic definitions of "close" and "completely cloudy sky"; that is, not only do the different algorithms apply different criteria to decide between clear and cloudy scenes, but also they are tuned using different test cases. The tuning of the algorithms constitutes a response to the problems of uncertainties in RS and TS and an attempt to improve the $\mathrm{CC}$ values obtained. The second problem is discussed further in Section 5.

\section{b. Inhomogeneous background}

Spatial variation of RS and TS within a small region requires better information on surface properties in order to recognize the presence of clouds in the scene. Coastlines and snow-covered land are common examples of scenes with highly variable surface properties which are more difficult to analyze even if the clear sky radiances are, roughly constant in time. This variability aggravates the confusion of clouds and clear sky because the radiances may form more than two statistical clusters (clear or cloudy) or may fail to form any cluster at all. The versions of the statistical methods and HB TH tested here are equivalent to the application of a single threshold value to a constant clear-sky radiance over the whole scene. The difficulty that this creates is illustrated by Fig. 11 showing two different types of scenes containing predominantly cloud covered ocean adjacent to clear land, cases for which the 

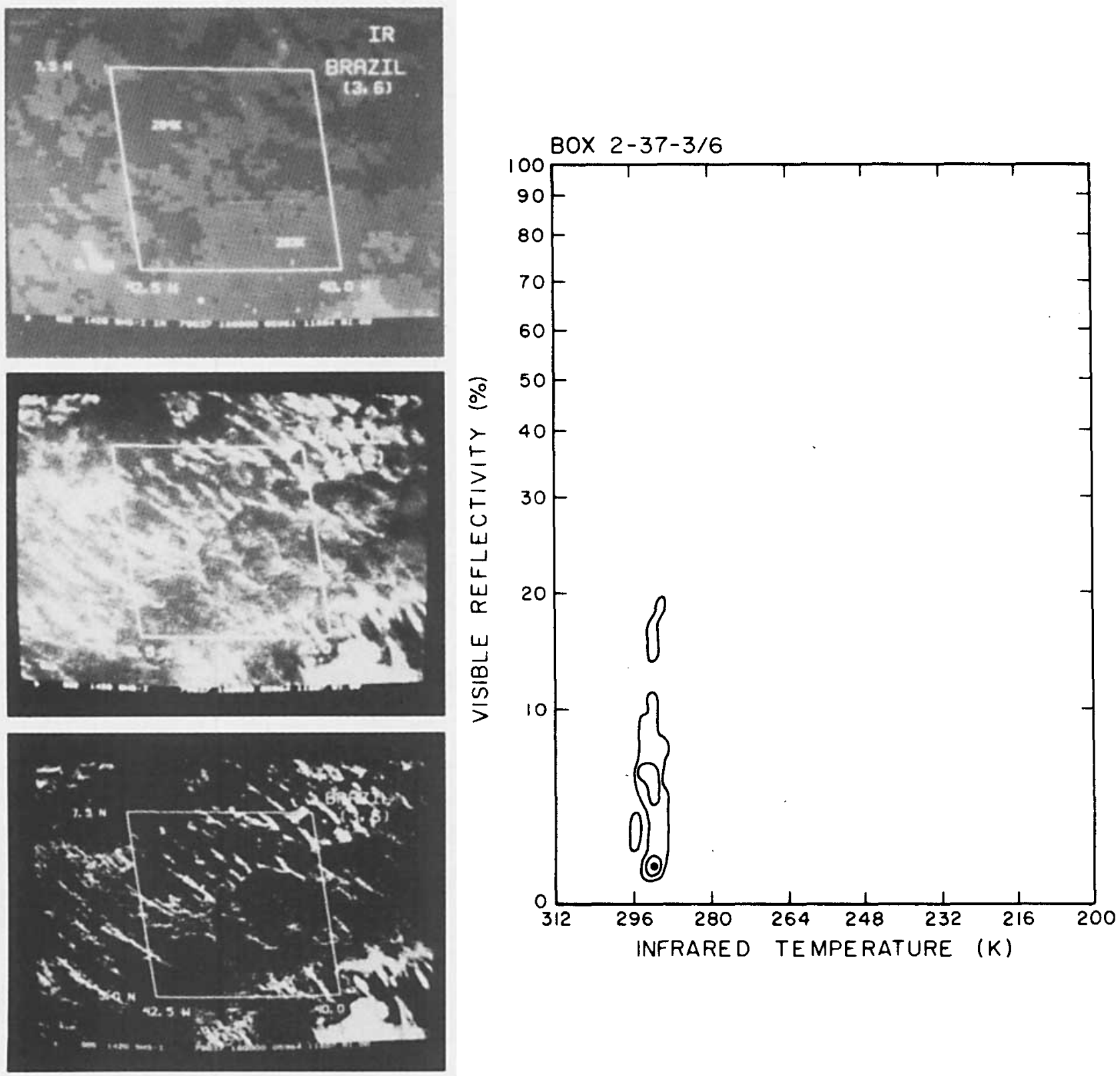

FIG. 8. Data for subregion 2-37-3/6 (see caption to Fig. 6). Note that IR contrast shown in upper photo is only $1 \mathrm{~K}$.

clear-sky radiances produce two distinct clusters. Where the cloud forms another distinct cluster in the histogram (Fig. 11a), DC HIS and AG HIS not only agree with each other on the value of $\mathrm{CC}$, but also agree well with the threshold methods. On the other hand, more broken cloudiness leads to a more complex histogram (Fig. $11 \mathrm{~b}$ ). This situation produces disagreement between the histogram methods and the HB TH method. (In this same scene, some of the thinner, low-lying cloud over ocean is missed by the RT TH method, whereas the IR TH method confuses some of the cooler mountains for cloud.)
An even more confusing case is illustrated in Fig. 12 for a region near the Great Lakes with partial ice cover and snow covered fields and forests. In this case the darkest area is also the coldest, representing clear skies over snow-covered forests in Canada. Because of a rapidly developing cyclone storm to the south, moving towards the east coast, the area is partially covered by cirrus/stratus which remains in a relatively constant location over a few days. Completing the confusion in this scene is the strong surface temperature gradient associated with the cloud front making the clear-sky temperature very similar to the apparent cloud top 

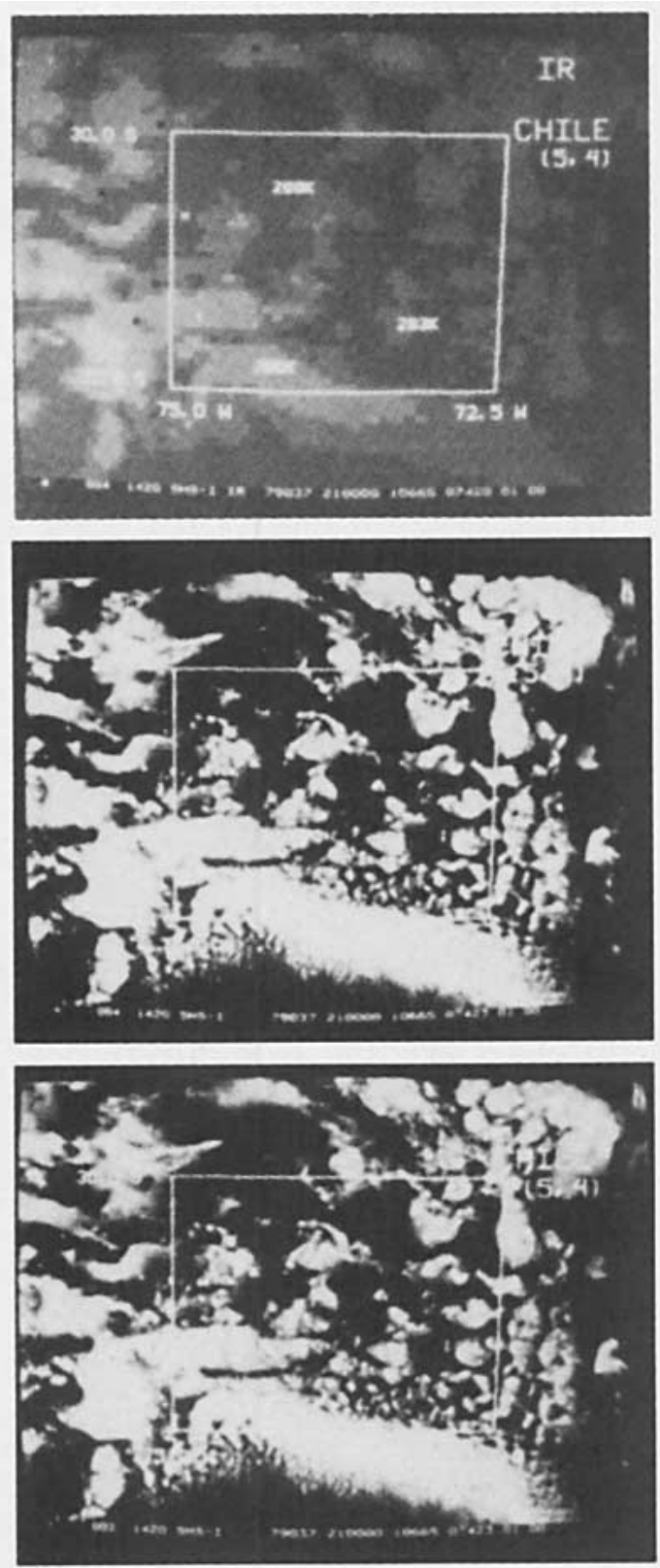

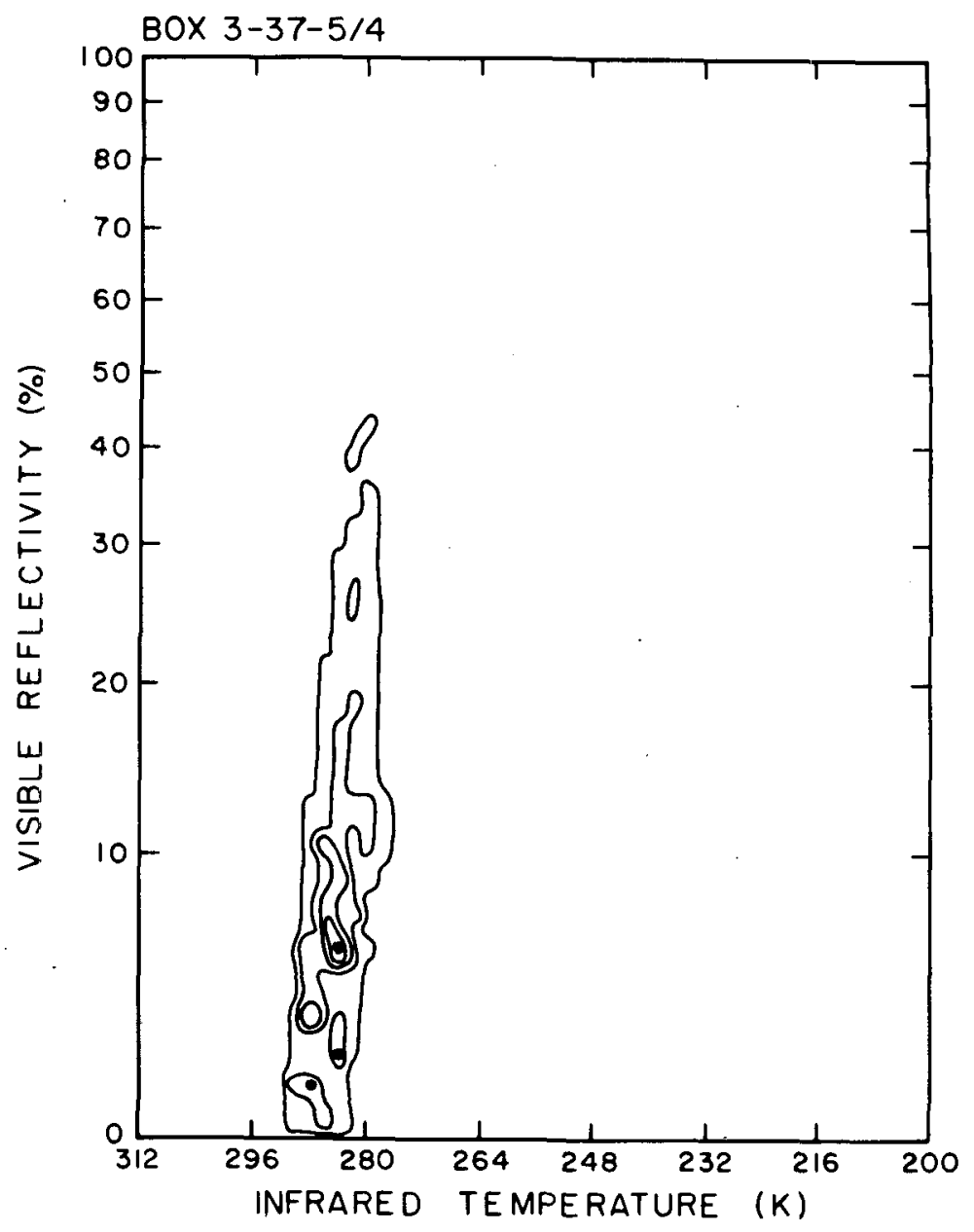

FIG. 9. Data for subregion 3-37-5/4 (see caption to Fig. 6). Note that IR contrast shown in upper photo is $6 \mathrm{~K}$.

temperature. Snow cover on open fields and partial lake ice cover produce some clear region visible brightnesses as large as that of the clouds. The histogram in Fig. 12 shows no distinct cluster associated with the clear or cloudy sky; this is confirmed by comparison of this histogram with those for regions to the northeast which are cloud-free and yet display similar histogram shapes. The results from the algorithms are quite scattered $(39 \pm 35)$; the best estimate of the actual cloud amount from visual inspection of the image data is $\sim 35 \%$.
Both of these cases stress the importance of using accurate descriptions of RS and TS in the analysis. Current statistical methods, which treat a large number of image pixels in an aggregate, have difficulty sorting complicated spatial patterns of radiances into cloud and clear regions because they are equivalent to the use of a constant threshold and clear-sky radiance over the whole region. The threshold methods tested in this study all use some form of time record analysis to obtain measures of the clear-sky radiance as a function of location. If surface and atmospheric properties re- 


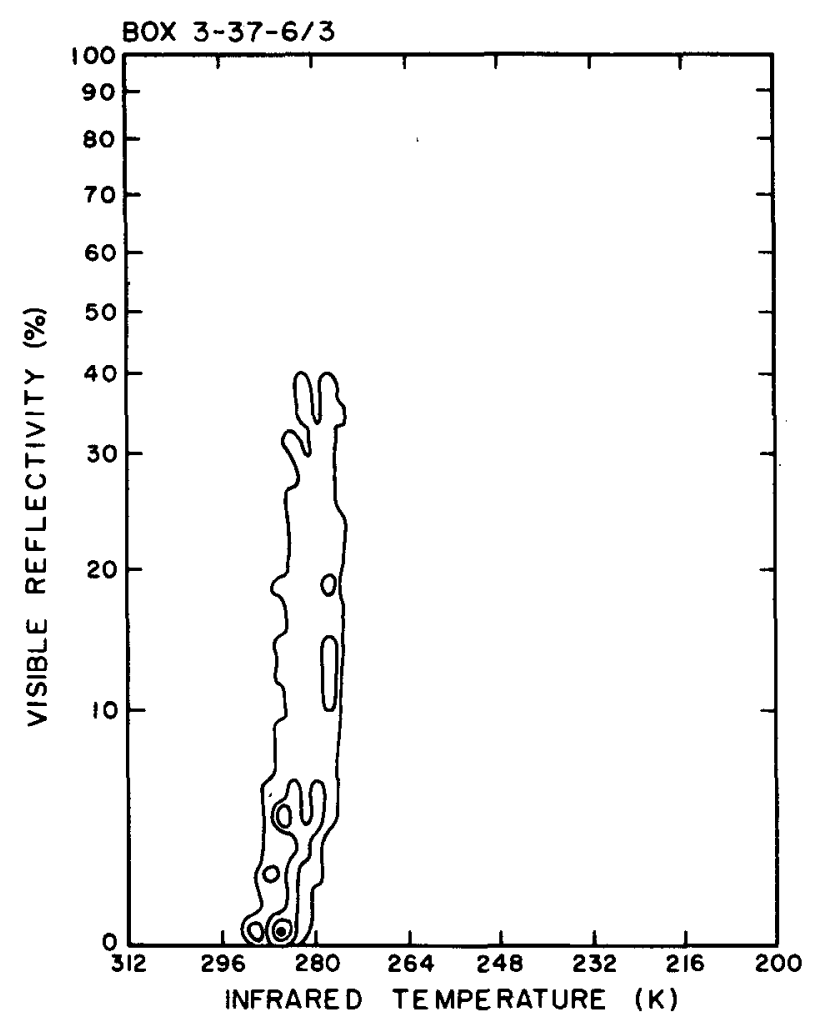

FIG. 10. Radiance histogram for subregion 3-37-6/3 (see caption to Fig. 6).

main relatively constant in time, then such an analysis can provide the detailed, pixel-by-pixel distribution of RS and TS. With this information the threshold methods can treat image pixels individually and are better able to handle these complex cases. A recent test of this time compositing approach with a histogram method shows that its results are also improved when the complicated surface clusters can be described (Desbois and Seze, 1984). In principle, then, all cloud algorithms can handle spatially complex scenes if accurate determinations of RS and TS are available and this information is utilized in the analysis.

\section{c. Time varying background}

The last case discussed above actually represents one of the most difficult classes of partially cloudy scenes to analyze correctly, since the surface properties are not only spatially complex but also varying rapidly in time. Although the use of clear sky values derived from satellite time composites can, in principle, account for the spatial variations of RS and TS, time variations in these quantities preclude a simple statistical analysis to obtain these values. A typical situation involves a rapidly developing winter cyclone. In only $24 \mathrm{~h}$, surface temperatures in regions which began the period ahead of a cold front, fall by $5-10^{\circ} \mathrm{C}$ with passage of the front;
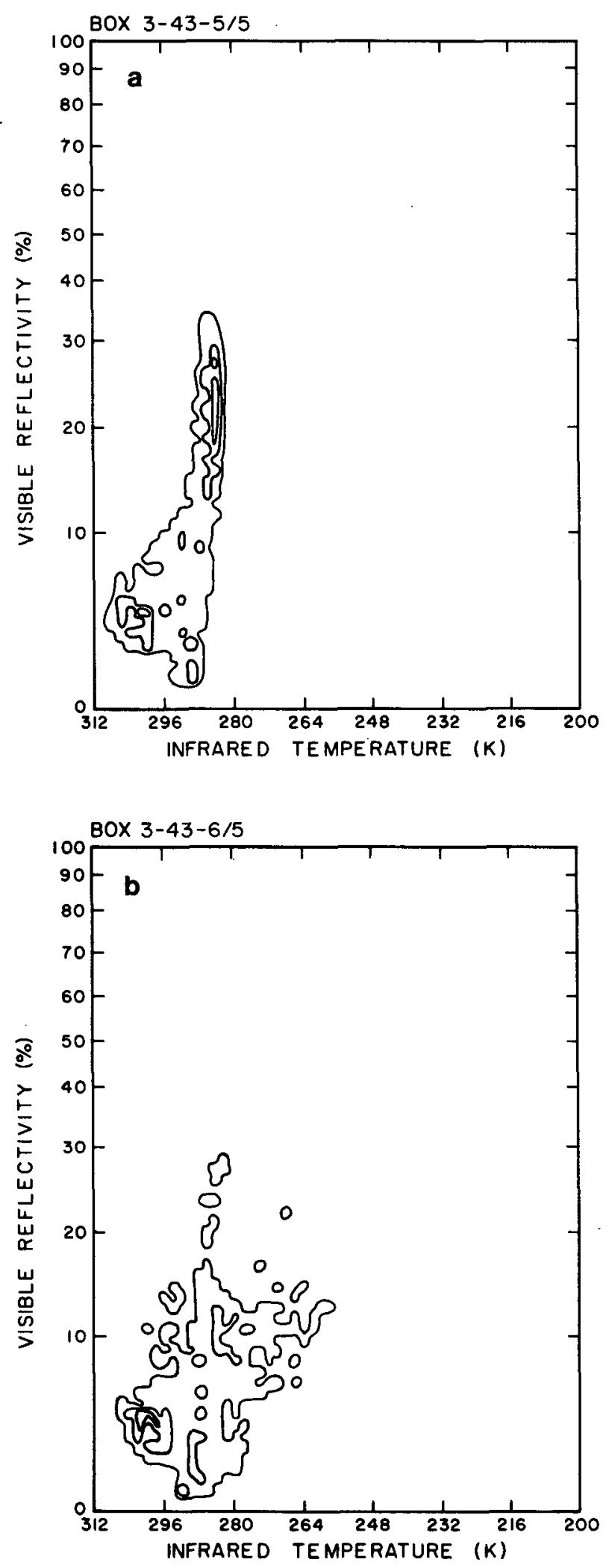

FIG. 11. Radiance histograms for subregion (a) 3-43-5/5 and (b) 3-43-6/5. 


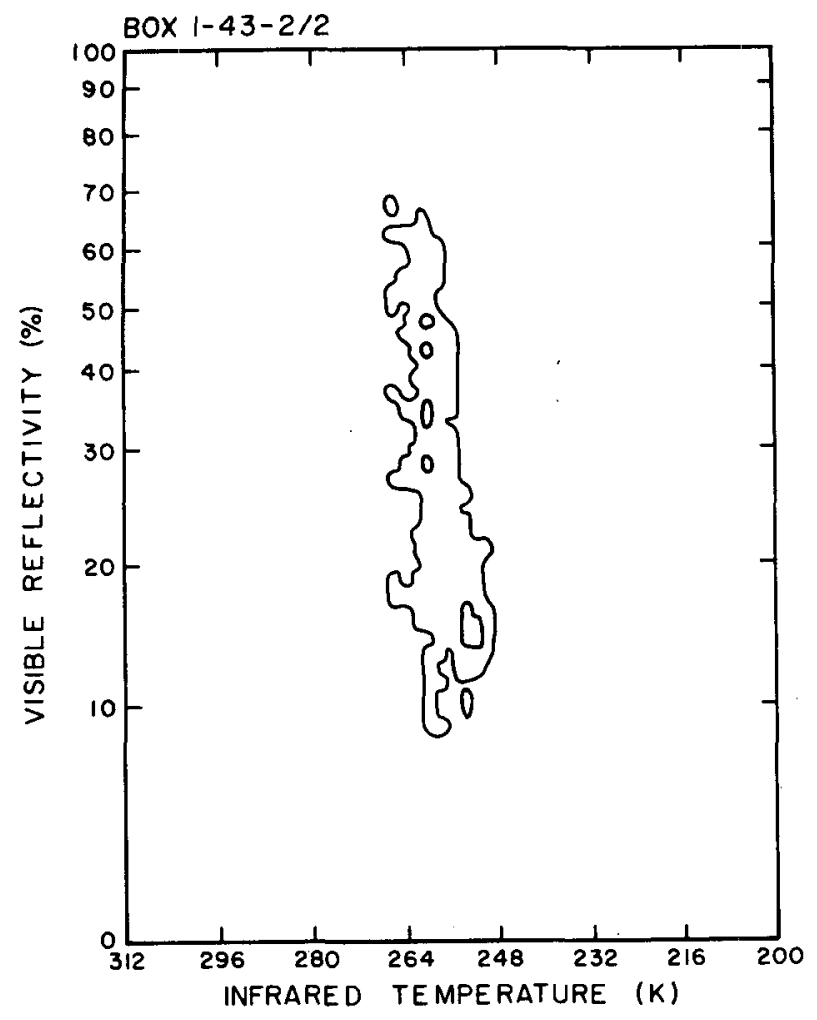

FIG. 12. Radiance histogram for subregion 1-43-2/2.

whereas temperatures elsewhere in the region remain relatively constant. The moving storm also adds to snow cover along the coast, around lakes, and on complicated patterns of field and forest. The complex spatial distribution of land albedo and surface temperature both change rapidly with time. These difficulties are exacerbated by the generally low contrast between winter clouds and snow covered land and explain the large range of $\mathrm{CC}$ values produced by all the methods in Region 1 (Figs. 2, 3, 4 and 5). The region encompassing the Great Lakes and areas adjacent to the St. Lawrence river valley are particularly difficult and are responsible for the largest disagreements, which are shown in Fig. 2 as points plotted against mean CC values between 30 and $60 \%$.

\section{d. Conclusions}

The discussion of specific cases in this section has focused on the most significant problems apparent in the intercomparison results. In addition to disagreements at the (broken) edges of large scale cloud systems, important problems are associated with particularly difficult cloud types, such as boundary layer cumulus or cirrus, or with complex and rapidly varying scenes like the wintertime U.S. and Canada. In other words, while a majority of cloud occurrences can be detected with current algorithms, if some information about clear sky radiance variations is used, the algorithm used by ISCCP must attempt to improve the treatment of these difficult cases. The specific cases discussed above (and in more detail in World Climate Program, 1984) suggest the following conclusions.

i) All algorithms benefit from accurate specification of clear-sky radiance values as a function of location and time. In fact, some of the disagreement between the algorithms discussed here can be attributed to the widely varying amounts of extra information used in these versions of the algorithms to define clear-sky radiance values, rather than to the methodological differences. Many of the difficulties with clouds over land, especially winter continents and mountainous terrain, might be removed with better information. Therefore, a major component of the ISCCP algorithm has to be the improvement of techniques to obtain clear-sky information from the satellite images and the collection and analysis of auxiliary data to improve clear-sky radiance specifications. Much of the improvement in cloud analysis anticipated from ISCCP will probably come from improvements in this part of the algorithm.

ii) Though all methods can benefit from improved clear sky information, the threshold method appears to be better developed at this time to utilize this kind of information, especially for spatially complex scenes. Little experience is available concerning use of this type of information with the statistical methods.

iii) Two important cloud types are especially difficult to detect: boundary layer cumulus and cirrus. These results suggest that detection of these types, not only requires accurate specifications of clear-sky radiances, but also very sensitive discrimination of radiance variations. Visible channel data seems indispensible for proper detection of the boundary layer clouds, even though this limits accurate results to daylight hours. Currently available statistical methods, which depend on cluster analysis of radiance histograms, seem less sensitive to small radiance variations than threshold methods (d'Entremont et al., 1982); the intercomparison results show these methods having more difficulty with these cases.

iv) Conclusion (iii) is tempered by two considerations. First, uncertainties in the specified clear-sky radiance and noise in the data, together with the reduced sensitivity which comes from spatial sampling of the data, may make use of very small threshold values inappropriate. Second, the overestimate of cloud fractional cover by counting pixels suggests choosing a somewhat larger threshold to neglect image pixels which are less than half covered. The latter problem is one of the design rationales for the statistical methods, but the same effect on $\mathrm{CC}$ values can be obtained by larger threshold values as well. Understanding the consequences of threshold choices is more straightforward for the threshold techniques than for the statistical methods. 
v) A second difficulty with identification of cirrus and boundary layer cumulus for current cluster analysis schemes is that these two cloud types do not always form a distinct cluster in the radiance histogram (see examples in World Climate Program, 1984). Threshold techniques, working with individual image pixels are not affected by this property. Nevertheless, the distinctive histograms produced by these clouds (see discussion in Section 5) do indicate potential additional information that can only be obtained by statistical methods. General techniques to recognize such patterns are not yet available, however.

vi) Detection of the difficult cloud types by a bispectral threshold technique is not completely straightforward. If clear-sky VIS and IR radiances are accurately known, the proper method would be to classify as cloud any image pixel which passes either threshold test. Neither RT TH or HB TH used this approach. Instead RT TH required a pixel to pass both threshold tests; but, since the IR test came first, RT TH results depend more on the IR test and they resemble IR TH more. Analysis method HB TH derives an IR threshold value, which is influenced by any clouds which fail a VIS threshold test, in order to obtain a better estimate of CC. However, as discussed in the specific case of boundary layer cloud, use of an IR threshold, however calculated, seems inappropriate.

vii) These conclusions are equivalent to saying that we have more experience with threshold methods than the newer statistical methods but that much improvement of threshold methods is still possible. The workshop participants selected a threshold method for the ISCCP algorithm, but did not choose any of the particular algorithms tested in this study. Rather, a new algorithm was designed (see Section 6) to overcome some of the deficiencies revealed by the intercomparison study.

\section{Cloud analysis}

After separating cloudy and clear scene radiances, further quantitative analysis of the cloudy sky radiances is required to meet the scientific objectives of ISCCP (Table 1, World Climate Program, 1982b). The large data volume produced by the high resolution radiometers on operational satellites requires compression by at least a factor of 1000 to make this analysis practicable, yet the effect of partially covered FOVs on determination of fractional cloud cover urges use of maximum resolution observations. Algorithm tests were directed towards study of some data compression schemes and of some techniques to account for partially covered FOVs.

\section{a. Data compression}

Since most operational radiometers produce much higher resolution visible channel data than IR channel data, consideration of data compression involves both the relation between the two channels and overall reduction of data volume. ISCCP objectives call for retrieval of those cloud properties which affect Earth's radiation budget; therefore, regardless of the way that partially covered FOVs are handled, the basic data for quantitative analysis of cloud optical properties are spectral radiance measurements of identical scenes. Since the ratio of VIS to IR resolution is very different for the available radiometers, no consistent method of utilizing the higher visible resolution has been developed. Hence, the first step in data compression is averaging of visible radiance measurements to produce a visible image pixel matched to the lower resolution IR image pixel.

Further data compression can be obtained by more degradation of spatial resolution either by averaging or sampling. (A few more sophisticated compression schemes were discussed by the workshop participants, but no proposals for recovering higher resolution cloud results from such methods were available.) The pilot dataset was used to examine whether spatial averaging or sampling should be used to produce the additional data volume reduction. Each algorithm group repeated some of their analyses with radiance data averaged to $32 \mathrm{~km}$ resolution and sampled to $32 \mathrm{~km}$ spacing. All methods exhibited the same behavior: $\mathrm{CC}$ calculated by counting cloudy pixels systematically increased by $5-10 \%$ when the data are averaged (cf., Shenk and Salomonson, 1972), whereas average results with sampled data were almost identical to the full resolution results. These test results recommend that further data compression by spatial sampling is the best strategy for climatological data sets.

\section{b. Analysis of cloud cover}

All of the cloud algorithms discussed thus far determine fractional cloud cover by counting all pixels classified as cloudy. If clear-sky radiance values are accurately known, then all algorithms can be tuned to detect all image pixels containing any amount of cloud; however, for broken clouds with individual cloud elements smaller than the radiometer FOV, this approach leads to an overestimate of fractional cloud cover. Both threshold and statistical methods can also be tuned to classify cloudy pixels, with lower partial coverage of the FOV, as clear to compensate for this effect; but, as the results in Section 4 show, not only is the required tuning dependent on the cloud types and size distribution, but also such tuning can cause the algorithm to miss certain cloud types altogether. The climatological importance of these difficult cloud types requires the ISCCP algorithm to detect their presence, but the problem of what cloud fraction to assign remains.

Some of the case studies discussed in Section 4 were designed to test whether any of the statistical methods 
showed any improvement in cloud amount determination over that of the (low resolution) threshold methods. As mentioned, the best "truth" for these tests was taken to be VIS TH applied to $1-\mathrm{km}$ resolution data over oceans. The VIS TH results were tuned by visual inspection of raw data images (with several exposures) and concurred in by the workshop participants. Pixel counting at $1 \mathrm{~km}$ resolution was deemed to give cloud amounts closer to actual values.

The methods tested were: (1) HB TH, (2) DC HIS (global), (3) DC HIS (local), (4) AG HIS, and (5) AG HIS (modified). Methods HB TH, DC HIS and AG HIS are tuned as described in Sections 3 and 4. Analysis method DC HIS (local) represents a reanalysis of the same data by DC HIS using cloud cluster classifications obtained from histogram analysis of each individual grid box rather than of the whole region. AG HIS (modified) attributes radiance variation about peaks in the histogram to two causes: (i) within one standard deviation the variation is assumed to be due to cloud optical property variation, whereas (ii) radiance variations between and further from cluster centers are caused by partial coverage of FOVs by two different "surfaces," either clear sky and cloud or two different clouds. Whereas before pixels between $\sigma$ and $3 \sigma$ distance were divided among clusters, in this version of the method, the pixels lying outside the cluster centers are assigned fractional cloud cover using a linear relation among radiances, coverage and the optical properties defined by the two clusters to optimize the match to the observed VIS and IR radiances. That is, $L=L_{1} f_{1}+L_{2}\left(1-f_{1}\right)$, where $L, L_{1}$ and $L_{2}$ are the pixel radiance and the cluster-mean radiances, respectively, and $f_{1}$ is the fraction of type 1 present.

Table 4 summarizes the results of the test for 11 ocean grid boxes by displaying the differences between each algorithm and VIS TH $(1 \mathrm{~km})$, the mean differ- ence and the standard deviation of these differences. Although the precise numerical values of the mean and standard deviation of differences are dependent on the set of cases selected, the table illustrates the conclusion that no method shows a consistent improvement relative to the other methods. Indeed the low resolution threshold results, also shown in Table 4, are similar to the statistical results, with only low resolution VIS TH showing a slight systematic difference. The conclusion, that all of these methods, many working with lower resolution data, obtain values of $\mathrm{CC}$ as good as those obtained by VIS TH applied to higher resolution data, is not completely warranted. The large standard deviation and the discussion in Section 4 suggest rather that the most important source of error for current algorithms is improper identification of clear sky radiances, even for simple ocean cases.

Two other methods have been proposed to improve fractional cloud cover determinations. The IR spatial coherence method (Coakley and Bretherton, 1982; Coakley and Baldwin, 1984) uses the spatial variability of the IR radiance to distinguish between partially covered FOVs and completely covered or completely clear FOVs in single-layered cloud systems. The radiance values of partially covered FOVs are intermediate between the clear and completely cloud covered values and are taken to be proportional to cloud fraction. The second method (Arking and Childs, 1985) is a variation of the modified AG HIS method discussed above which determines fractional coverage of image pixels by 1) forming the two-dimensional histogram of cloud optical thickness and cloud top temperature calculated from the radiances assuming complete coverage of all FOVs and then 2) minimizing the dispersion of clusters in this histogram by varying cloud fraction. Limited comparisons of results from both of these methods to those obtained from a pixel counting technique (RT

TABLE 4. Differences between algorithm results and VIS TH $(1 \mathrm{~km})$ results for 11 ocean cases. Values in parentheses under VIS TH represent results excluding two extreme values.

\begin{tabular}{|c|c|c|c|c|c|c|c|}
\hline $\begin{array}{c}\text { Box } \\
\text { number }\end{array}$ & HB TH & AG HIS & $\begin{array}{l}\text { AG HIS } \\
\text { (mod) }\end{array}$ & $\begin{array}{l}\text { DC HIS } \\
\text { (global) }\end{array}$ & $\begin{array}{c}\text { DC HIS } \\
\text { (local) }\end{array}$ & $\begin{array}{l}\text { VIS TH } \\
\text { (8 km) }\end{array}$ & $\begin{array}{c}\text { RT TH } \\
(8 \mathrm{~km})\end{array}$ \\
\hline $1-37-5 / 5$ & 22 & 24 & 13 & 16 & & 5 & 19 \\
\hline $1-37-8 / 6$ & 5 & 12 & 11 & -14 & 7 & 3 & -5 \\
\hline $1-37-8 / 7$ & -5 & 33 & 27 & -39 & -38 & 12 & -26 \\
\hline $2-37-3 / 6$ & 17 & -13 & -5 & -15 & -7 & 1 & -14 \\
\hline $2-37-4 / 6$ & 1 & -5 & 0 & -26 & 4 & 6 & -15 \\
\hline $3-37-2 / 4$ & -1 & 0 & 0 & 5 & 5 & 1 & 8 \\
\hline $3-37-3 / 4$ & -7 & -11 & -7 & 17 & & 2 & 27 \\
\hline $3-37-4 / 4$ & -20 & -15 & -18 & 0 & 6 & -12 & 5 \\
\hline $3-37-5 / 4$ & -8 & 2 & 7 & 13 & 13 & -5 & 16 \\
\hline $3-37-6 / 3$ & -26 & -35 & -34 & -10 & -11 & & -25 \\
\hline $3-37-6 / 4$ & -2 & -12 & -12 & -2 & -2 & & -8 \\
\hline Mean & -2 & -2 & -2 & -5 & -3 & $1(2)$ & -2 \\
\hline $\begin{array}{l}\text { Standard } \\
\text { deviation }\end{array}$ & 13 & 18 & 16 & 17 & 14 & $6(3)$ & 17 \\
\hline
\end{tabular}


TH), all applied to 4-km resolution AVHRR data, show that cloud amounts are generally reduced and suggest some agreement between the two methods (see World Climate Program, 1984).

Several issues remain to be explored, however. All of the methods discussed in this section attempt to improve fractional cloud cover determinations using some statistical property of the radiance spatial distribution; however, they also assume some constant radiative property and, more critically, assume that some particular radiance value represents a completely cloud covered image pixel. Broken cumulus clouds, for which the FOV problem is most severe, do not always oblige by providing any completely cloud covered pixels. Figure 13 illustrates the fundamental dilemma by showing the interrelation between cloud fraction, optical thickness, and top temperature for two cases: many possible solutions can explain one VIS and IR radiance observation. Unique identification of which variables are responsible for the variation in the observed radiances may depend on the correct identification of different cloud types and is not currently possible on a global basis. Much more study of these and other suggestions for treating partially covered FOVs is required.

\section{c. Analysis of diurnal variations}

The main focus of the pilot study was on the analysis of cloud amount using both VIS and IR radiance measurements. However, the goal is to design an algorithm which obtains the complete diurnal variation of clouds.
For nighttime observations, this requires the algorithm to be applied to IR radiance data, alone, for global results. This produces two difficulties for analysis of the diurnal variation of clouds. First, all algorithms become, to some degree, less capable of detecting lowlying clouds, particularly over land where the surface temperature (and clear-sky IR radiance) is generally closer to the cloud top value and undergoes rapid and spatially complex variations (see discussion in Minnis and Harrison, 1984a,b). If the surface reflectivity is known, VIS radiance measurements during the day allow for a more sensitive detection of low clouds. As a consequence, proper evaluation of the diurnal variation of low cloud cover is difficult. Second, with only IR information available, the quantitative analysis of cloud optical properties is constrained in a different fashion. Optically thin clouds, in particular, cannot be uniquely distinguished from warmer, optically thick clouds; thus, the diurnal variation of cirrus may not be reliably measured.

\section{ISCCP cloud algorithm design}

Figure 14 illustrates the ISCCP operational algorithm, which has four major components: 1) derivation of clear-sky radiance maps from satellite radiance and correlative data that correspond to each image, 2) application of radiance thresholds, 3) comparison of cloudy radiances to the cloud model radiances, and 4) histogram and diagnostic analysis of radiances and

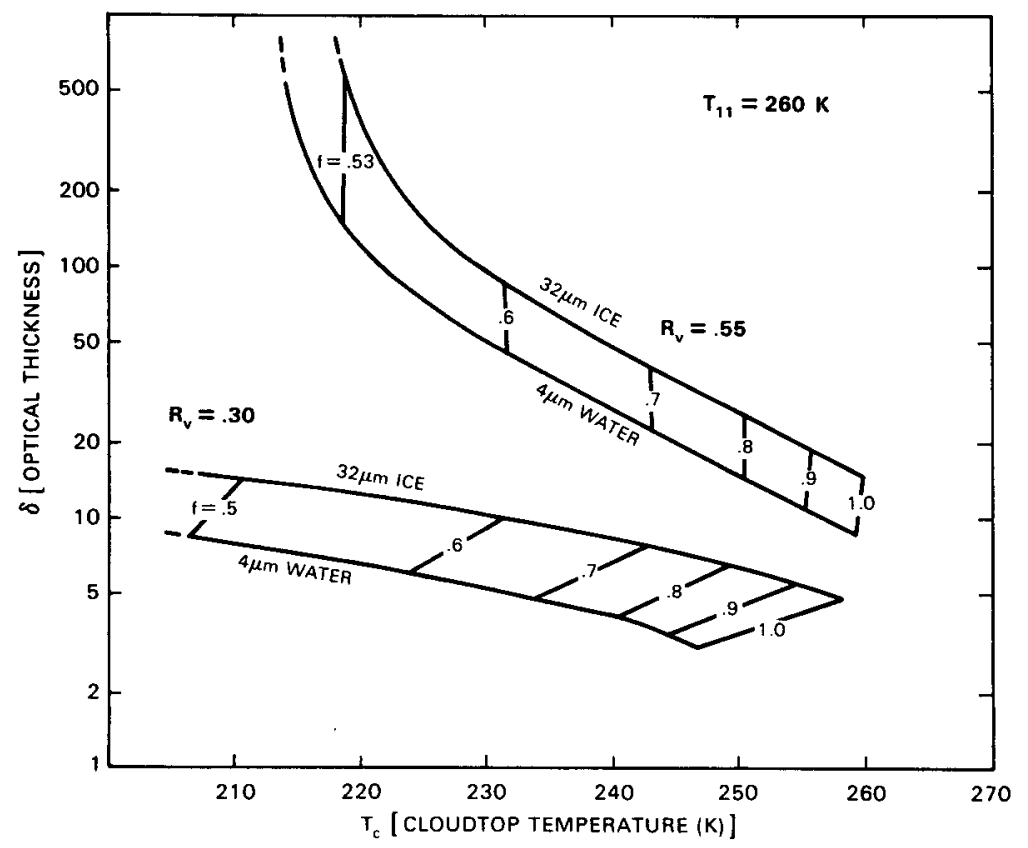

FIG. 13. Variation of VIS and IR radiances for cloud with fixed properties but covering variable fractions of the satellite radiometer FOV (from Arking). 


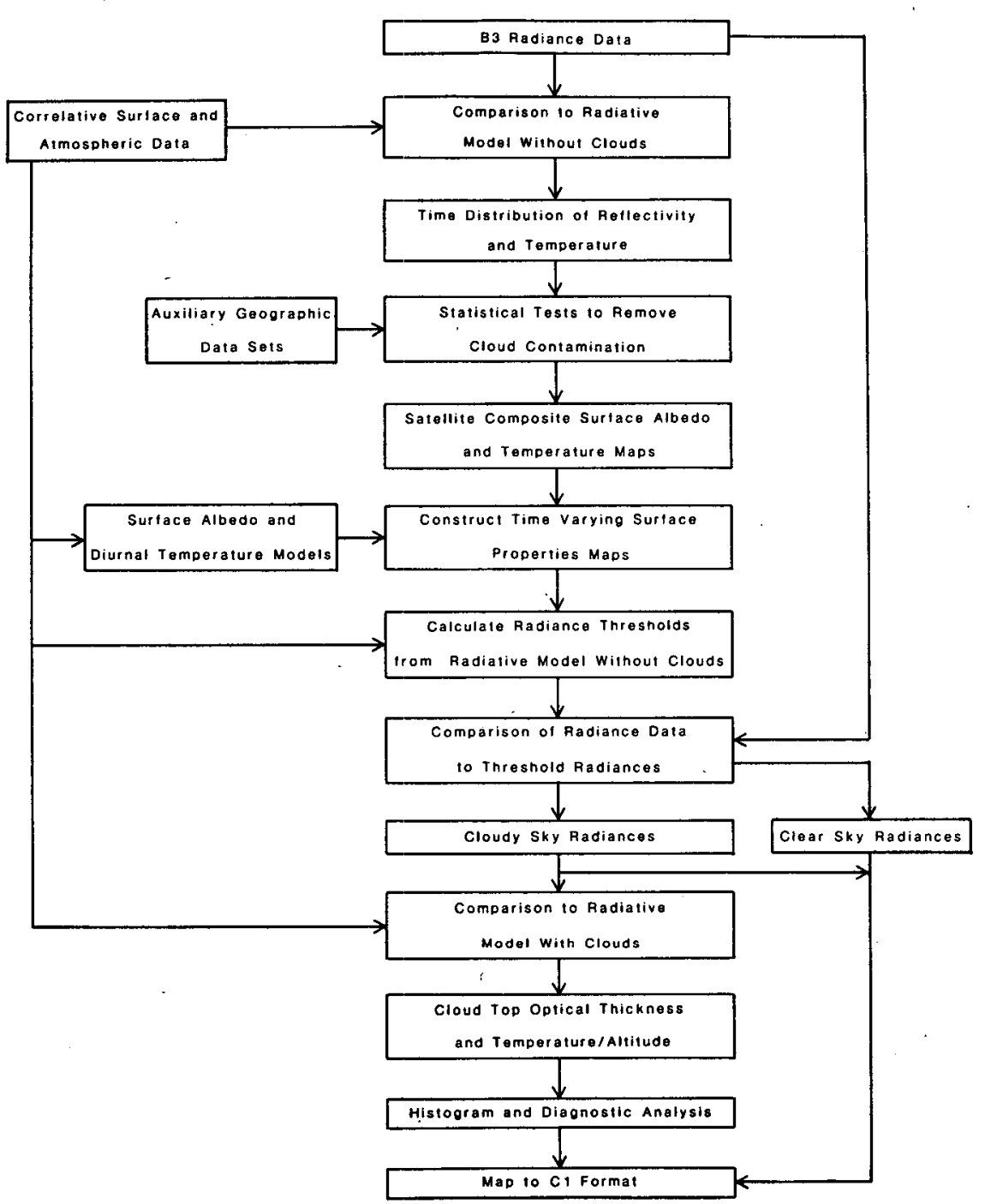

FIG. 14. Schematic of ISCCP operational algorithm. B3 data refers to reduced volume radiance data which have been normalized to the AVHRR response; correlative data refers to auxiliary data sets specifying surface and atmospheric properties. $C$ data refers to basic output data from the analysis.

cloud properties. Each of these components is described below.

\section{a. Clear-sky radiance map}

Infrared and VIS clear-sky radiances are a function of atmospheric and surface radiative properties, which in turn vary with location and time. Analyses of the satellite radiance data can obtain measures of the clearsky radiances, though the removal of cloud contamination is not complete (the converse of the cloud detection problem discussed in Section 4) and the space- time coverage is incomplete. Several other available satellite and conventional weather data sets provide information on the state of the atmosphere and surface, but these data sets do not provide complete spacetime coverage either. The approach adopted for ISCCP is to combine several data sets and analysis techniques to obtain a complete description of the clear-sky radiance distribution. No single step in the analysis is expected to be perfect or complete; subsequent steps check and correct the results of prior steps. The data and analysis steps are arranged in a hierarchical fashion so that less accurate or less direct results are used last; 
thus, correlative data from other sources are used primarily to fill gaps in satellite observations of clear-sky radiances or to resolve ambiguous situations. The three basic steps in the analysis, which were suggested by the algorithms in the pilot study are: classification of satellite images, compositing satellite images, and filling and interpolation using correlative data.

Each of these steps focuses on a different aspect of the complete clear-sky radiance distribution because the space-time variations differ between spectral channels and vary with surface type. Clear-sky IR radiances over the ocean are relatively constant in time and less variable in space compared to those over land. Clear sky VIS radiances are much more constant in time but more variable in space than clear-sky IR radiances. The behavior of both land and ocean is altered at high latitudes by the presence of snow and sea ice. Thus the clear sky radiance analysis differs somewhat over each of four surface types: land, snow/ice-covered land, ocean and ice-covered ocean.

The first step is classification of the satellite IR images into cloudy and "clear" categories based on the characteristic of the spatial distribution of radiances that, within many subregions approximately $100-200 \mathrm{~km}$ square, some clear sky is present and the cloud top temperature is very different from clear sky. Thus any pixels found to be much colder than the warmest pixel in that subregion are labeled cloudy. In general, this crude threshold properly identifies most high and middle level clouds constituting about one-third of the image and about half of the total cloud; however, in some areas with low cloud or in some regions completely covered by layered clouds, no pixels are classified as cloudy. Next each pixel is classified by the time variation of the IR radiance: any pixel that is much colder than on the previous day or the following day is labeled as cloudy. This step works for many clouds that have top temperatures much colder than clear sky and move or develop significantly in one day. Again, most middle and high clouds associated with synoptic or mesoscale storm systems, as well as some broken cumulus clouds, are detected. About one-third of the image is classified as cloudy by this step, but much of this cloud was also identified in the first step. The total detected by either step is a little more than half of the total cloud present; the total detected by both steps together is about twothirds of the total cloud. Image classification thus serves to identify the "obvious" clouds in IR radiance images.

The second step, compositing, examines the time and space variations of radiances in a different way. The cluster analysis methods (Desbois et al., 1982; Simmer et al., 1982) and the method of Coakley and Bretherton (1982) all make use of the general observation that clear regions exhibit smaller spatial variations in IR radiance than do broken cloud regions. However, some low cloud types, particularly the marine stratocumulus studied by Coakley and Bretherton, can also exhibit small spatial variance when cloud cover is complete. Trade-wind cumulus at very low levels can also exhibit small spatial variances even though cloud cover is much less than complete. Over ocean, where time variations of both VIS and IR clear sky radiances are small, some cloudy radiances confused with clear in the first step can be recognized by their variation in time, as suggested by Minnis and Harrison (1984a) and Rossow $e t$ al. (1985). Thus the frequency of occurrence of the radiance values associated with clear sky is generally higher when a small spatial domain is examined over time. The extremum of the radiance distribution, usually associated with clear sky (minimum reflectance, maximum brightness temperature), is too sensitive to unusual circumstances, but should generally be close in value to the average over a small space and time domain if that region is clear of clouds. The compositing step checks for this condition using only the radiance values classified as "clear" by the first algorithm step. The results of this step can reinforce or contradict that of the first step, especially over land where clear sky IR can vary rapidly in time. Contradictions are labeled as "confused."

Statistical studies of the spatial (Rossow et al., 1985) and temporal (Minnis and Harrison, 1984a) correlations of clear-sky IR radiances suggest that some (limited and cautious) interpolation can be done to obtain more coverage of their distribution. In particular, IR radiances in confused subregions which are similar to radiances in adjacent clear subregions provide a clue to the proper value in that location.

At this stage, there are some locations or times for which clear-sky radiances have not been obtained because of persistent cloud cover, rapidly varying (i.e., confusing) surface properties or ambiguities in the previous analysis steps. The third step in the analysis is to compare the incomplete clear sky measurements obtained from the satellites with colocated conventional observations. This comparison (by region) is used to provide a statistical relationship used to translate the correlative data into clear sky radiances as measured by the satellite (see Rossow et al., 1985) and to fill any gaps in the satellite analysis. The advantage of this approach is that radiometer calibration effects and radiative model deficiencies are nearly eliminated by removing any bias between satellite and correlative data. Furthermore, these values are generally only used in regions which are mostly cloudy and, therefore, do not affect the detection step very strongly.

\section{b. Threshold application}

The data in a particular image are now compared to the clear sky values modified by a threshold amount, $\Delta R$ and $\Delta T$. These threshold amounts are meant to account for three distinct effects. The first is the effect on the analysis of measurement and processing errors 
in the satellite radiance and the correlative data used in the analysis. The second effect is that of the uncertainties in the clear-sky radiances obtained from the analysis process outlined above, inaccuracies in the radiative model, and lack of adequate information to specify correct radiative properties. The radiative model problems are associated with uncertainties in angular dependence of surface reflectivities, surface emissivity variations, and variations in atmospheric aerosols not accounted for in the clear-sky radiance map construction. (Indeed, large changes in aerosol, such as Saharan dust outbreaks, will appear as clouds in this climatology.) The third effect is that caused by very low, very small scale broken or thin clouds which make only marginal changes in the measured radiances. The first two effects are equivalent to noise in the cloud detection measurement (radiance minus clear sky value) while the third effect represents a very weak signal. Selection of the threshold value is equivalent to requiring that the detection signal be greater than the detection "noise."

The actual application of the thresholds to decide the presence of cloud will make best use of available information. Thus a bispectral method for daytime data should allow detection of clouds by one spectral channel that are nearly indetectable in the other channel. Cirrus are more difficult to detect in VIS radiances than IR, while low-lying ocean trade wind cumulus are more difficult to detect in IR radiances. The bispectral threshold logic used is to label an image pixel cloudy if either radiance exceeds the threshold. At night with only IR radiances available, detection of low clouds will be more uncertain. (In all cases the channels detecting the cloud will be recorded.)

An estimate of the uncertainty in cloud amount can be obtained by counting the number of image pixels with radiance values near the threshold value. Examination of the pilot study data in radiance histogram form shows that the presence of broken clouds or more complicated surface properties in a region, which caused trouble for all the algorithms, is usually reflected in the presence of large numbers of pixels near the (proper) clear-sky radiance values or confused with the clear sky values. Consequently, counting the number of pixels near the threshold values provides a fair estimate of the uncertainty in the cloud amount.

\section{c. Calculation of cloud radiative parameters}

The radiance values associated with cloudy scenes by the threshold tests are next compared to model calculated radiances to determine cloud optical thickness and cloud top temperature. The radiative and cloud models for this comparison define a parameterization of the processes which determine the radiances measured by satellite radiometers. Cloud effects on satellite- measured radiances are parameterized in terms of three variables: cloud cover fraction ( 0 or $100 \%$ in each pixel), cloud optical thickness and cloud top temperature. Since the parameterization of atmospheric and surface effects on radiances, employed for these calculations, is the same one used to infer the clear-sky radiances, the resulting cloud properties are just those cloud properties (assuming complete coverage of the pixel) which are required to explain the differences between clear sky and observed radiances (cf. Reynolds and Vonder Haar, 1977). The complete set of results inferred from the satellite and correlative data is thus tied together by the model as a self-consistent radiative description of the surface-atmosphere-cloud column at each location and time. All of the parameter values used in the analysis are therefore reported as part of the output.

The cloud model selected for the analysis is a planeparallel cloud with an effective (cross-section weighted) mean droplet radius of $10 \mu \mathrm{m}$ (Hansen and Pollack, 1970). The cloud effects on VIS radiances are parameterized in terms of the cloud optical thickness calculated for conservative scattering in a single cloud layer embedded in a gaseous atmosphere. Ozone absorption in the stratosphere is accounted for by increasing the observed radiance by an amount equivalent to two transmissions through the ozone layer. The land surface reflectivity uses two different bidirectional functions for vegetated and unvegetated land surfaces (e.g., Kimes, 1983) tied to the satellite VIS radiance composite maps. An ocean reflectivity model obtained from satellite observations by Minnis and Harrison (1984a) is used.

The IR radiance model calculates the emission of radiation from a surface with unit emissivity and a single layer cloud with unit emissivity through an atmosphere with opacity determined by a water vapor profile. (Use of unit emissivity for the surface is also assumed to retrieve the satellite surface temperature; consequently, only a small error remains because of a small reflection of downward radiation by the surface.) The atmospheric emission is also calculated using the specified temperature and humidity profiles. If cloud optical thickness is measured (daytime), the cloud-top temperature is also calculated for the corresponding emissivity value. Cloud-top temperature and the atmospheric temperature profile are used to calculate cloud-top pressure.

\section{d. Histogram and diagnostics}

Examination of the radiances and cloud optical parameters on a regional scale employing histogram techniques is performed to check the consistency of the results overall and to provide information which classifies the surface and cloud types present. As illustrated in the pilot study results (see World Climate 
Program, 1984), the structure of the radiance histogram seems diagnostic of different cloud morphologies. Results of an experimental classification scheme will be reported.

Several types of quality labels will be reported with the results to document the choices made by the analysis program, to evaluate the consistency of the results, and to warn of difficult situations which give less reliable values. In the latter category are retrievals performed under temperature inversion conditions, over snow or ice covered surfaces (especially for geometries which cause thinner clouds to be darker than the surface) and in sun glint geometries. These quality labels allow for later consistency checks to verify that the less reliable retrievals do not introduce any bias in the results.

\section{e. Reported variables}

Although the analysis map grid used for the diagnostics is an equal area map, necessary to preserve proper statistical uniformity over the globe, tests have shown that remapping of such results to other projections does not affect the statistical properties of the results (Rossow and Garder, 1984). Thus, ISCCP cloud data can be easily remapped to other commonly used grids, such as the square latitude-longitude format. The spatial resolution of the results is equivalent to $2.5^{\circ}$ near the equator. Results will be presented for every 3 $\mathrm{h}$ over the whole globe and averaged over calendar months. The variables reported for each grid box are the fractional cloud cover and error estimate and the mean and variance of cloud optical thickness, top temperature and top pressure for (at least) six cloud types: total, low, middle, high, cirrus and deep convective. Each of these types is defined by cloud top pressure and/or optical thickness as follows: low cloud top pressure, $\mathrm{PT} \geqslant 700 \mathrm{mb}$; middle cloud top pressure, 700 $>$ PT $\geqslant 500 \mathrm{mb}$; high cloud top pressure, $500 \mathrm{mb}$ $>$ PT. The pressure levels correspond roughly to 3 and $6 \mathrm{~km}$ altitudes. Cirrus and deep convective clouds are defined (during the day) to be high clouds with optical thickness, $\tau \leqslant 6$ and $\tau>32$. The former definition is selected to insure that the clouds identified as cirrus have properties which lead to positive radiative feedback on climate. Higher clouds could be somewhat thicker and still provide a small positive feedback.

Also reported are the mean and variance of total and clear-sky radiances (for all available spectral channels), surface temperature, humidity and visible reflectivity, surface type and topography, atmospheric temperature and humidity profiles, and ozone column abundance. Additional information about the amount and kind of data used in the analysis and the quality labels are also included. On a somewhat larger regional scale, the histogram and other spatial distribution di- agnostics, plus surface and cloud type classifications, are reported. Monthly averages of these results will include a mean diurnal cycle and other time distribution diagnostics.

\section{f. Recommendations for future research}

The research component of ISCCP is concerned with validation of the cloud climatology produced by the algorithm described above; however, validation has two different, but related, objectives. The first is to clarify the interpretation of the climatology dataset so that it can be used to study cloud behavior and to improve the treatment of clouds in climate models. The focus of this type of study is on understanding the atmosphere and how to model it. The second objective is to confirm the cloud parameterization used in the analysis of satellite data and to improve analysis techniques. Progress towards both of these objectives requires improved understanding of the interaction of radiation and clouds. The recommendations from the Workshop participants concern the second objective.

Future work to improve cloud algorithms should focus on four areas: development of histogram and other statistical methods, development of multichannel radiative transfer methods, study of cloud morphology statistics, and investigation of methods to infer additional cloud properties from satellite observations. Several statistical approaches have been proposed to obtain better cloud cover values than possible with pixel counting; however, the pilot study results suggest that these methods may work only when the clouds behave as assumed in the technique. Proper exploitation of these methods may require identification of cloud or scene type before application to account for the different statistical behavior that seems related to different cloud types. If further study of cloud property spatial statistics shows a recognizable pattern for each cloud morphological type, then these methods may be used both to classify cloud types and to calculate more accurate cloud cover.

Another way to resolve the dilemma of recognizing the difference between radiance changes due to cloud cover variations and those due to optical property variations is to employ at least one more independent radiance measurement to retrieve a unique set of cloud parameters. Several existing radiometers have other spectral channels which could be tested for this purpose. Whether pixel-by-pixel retrievals or histogram methods are to be employed probably depends on the spectral channels used; both types of multichannel techniques should be tried. All available spectral channels are saved as part of the ISCCP radiance dataset.

The structures exhibited by the histograms are, at least in part, an expression of the fact that clouds or cloud fields are generally large-scale features controlled 
by the larger amplitude motions in the atmosphere. Even convection occurs in mesoscale to synoptic scale complexes. Nevertheless these large features are rich in the smaller scale structures which allow identification of cloud types (which were originally defined by morphology). Further study of histogram and other statistical descriptions of cloud morphology on all (accessible) space and time scales could lead to a climatology of dynamics-cloud physics interactions which would contribute significantly to understanding of cloud effects on climate. While current climate models may obtain plausible cloud cover amounts, they may do so for the wrong reasons. A climatology of cloud morphology and frequency of occurrence of cloud type would challenge the details of cloud parameterizations.

The improvement of understanding of clouds in general and of satellite observations of clouds in particular, gained from all of the above studies, leads naturally to study of what other cloud properties can be observed from space. Of particular importance is study of the relation of the cloud properties derived in ISCCP to the effect of clouds on the planetary and surface radiation budgets. As stressed in this paper, the limitations of the data force all algorithms to use models which parameterize cloud effects on radiance measurements in terms of a small number of variables. More sophisticated observations will be required to improve and expand these parameterizations. Research in this area has two foci: 1) attempts to derive other properties from currently available data or to correlate other properties with the retrieved optical properties and 2) design of new instruments that can retrieve new cloud properties. Of particular importance in the list of possible new variables are the cloud base altitude, cloud vertical structure and precipitation amounts. Systematic monitoring of these quantities from space, when added to the quantities which are the focus of ISCCP, would improve the study of the global surface radiation budget, cloud effects on radiative flux divergence in the atmosphere, and the hydrological cycle. The latter two may not be complete without more accurate monitoring of water vapor from space, however. The emphasis on developing techniques for satellite observations seems necessary to obtain a global description of these phenomena on our planet.

Acknowledgments. The participants (see Appendix A in World Climate Program, 1984) in the four workshops contributed many useful ideas and provided stimulating discussions of the concepts discussed in this paper. All data sets were supplied by Dr. Robert A. Schiffer, NASA Climate Program Manager and the ISCCP Project Manager for WMO. The large number of figures in this paper and the larger report were prepared by L. DelValle and J. Mendoza; the several versions of the manuscript were typed by A. Calarco and D. Smith.

\section{REFERENCES}

Arking, A., and J. D. Childs, 1985: Retrieval of cloud cover parameters from multispectral satellite measurements. J. Climate Appl. Meteor., 24, 322-333.

Chahine, M. T., 1982: Remote sensing of cloud parameters. J. Atmos. Sci., 39, 159-170.

Coakley, J. A., 1983: Properties of multilayered cloud systems from satellite imagery. $J$. Geophys. Res., 88, 10 808-10 830.

- , and F. P. Bretherton, 1982: Cloud cover from high-resolution scanner data: Detecting and allowing for partially filled fields of view. J. Geophys. Res., 87, 4917-4932.

- - and D. G. Baldwin, 1984: Towards the objective analysis of clouds from satellite imagery data. J. Climate Appl. Meteor., 23, 1065-1099.

Cox, C., and W. Munk, 1956: Slopes of the sea surface deduced from photographs of the sun glitter. Bull. Scripps Inst. Oceanogr., 6, 401-488.

Desbois, M., and G. Seze, 1984: Use of space and time sampling to produce representative satellite cloud classifications. Ann. Geophys., 599-606.

, - and G. Szejwach, 1982: Automatic classification of clouds on METEOSAT imagery: Application to high-level clouds. $J$. Appl. Meteor., 21, 401-412.

d'Entremont, R. P., R. S. Hawkin and J. T. Bunting, 1982: Evaluation of automated imagery analysis algorithms for use in the threedimensional nephanalysis model at AFGWC. USAF Environ. Res. Pap. No. 817, AFGL-TR-82-0397, 39 pp.

Garder, L., E. Kinsella, W. B. Rossow, A. A. Lacis and C. Brest, 1985: Global cloud properties from radiative analysis measurements. Part I: Methodology and cloud amount. J. Climate Appl. Meteor., (in press).

GARP, 1975: The Physical Basis of Climate and Climate Modeling. GARP Publ. Ser. No. 16, 265 pp.

GARP, 1978: JOC study conference on parameterizations of extended cloudiness and radiation for climate models. Oxford, GARP Climate Dynamics Sub-program, 154 pp.

Hansen, J. E., and J. B. Pollack, 1970: Near-infrared light scattering by terrestrial clouds. J. Atmos. Sci., 27, 265-281.

Harrison, E. F., P. Minnis and G. G. Gibson, 1983: Orbital and cloud cover sampling analyses for multisatellite earth radiation budget experiments. J. Spacecraft Rockets, 20, 491-495.

Hilsenrath, E., and B. M. Schlesinger, 1981: Total ozone seasonal and interannual variations derived from the 7 year Nimbus-4 BUV data set. J. Geophys. Res., 86, 12 087-12 096.

Kidwell, K. B., 1981: NOAA polar orbiter data (TIROS-N and NOAA-6) users guide. NOAA, U.S. Dept. of Commerce, 110 pp.

Kimes, D. S., 1983: Dynamics of directional reflectance factor distributions for vegetation canopies. Appl. Optics, 22, 1364-1372.

Minnis, P., and E. F. Harrison, 1984a: Diurnal variability of regional cloud and clear sky radiative parameters derived from GOES data. Part I: Analysis method. J. Climate Appl. Meteor., 23, 993-1011.

$\longrightarrow$, and - 1984b: Diurnal variability of regional cloud and clear sky radiative parameters derived from GOES data. Part II: November 1978 cloud distributions. J. Climate Appl. Meteor., 23, 1012-1031.

- , and $-1984 \mathrm{c}$ : Diurnal variability of regional cloud and clear sky radiative parameters derived from GOES data. Part III: November 1978 radiative parameters. J. Climate Appl. Meteor., 23, 1032-1051.

Norton, C. C., F. R. Mosher, B. Hinton, D. W. Martin, D. Santek and W. Kuhlow, 1980: A model for calculating desert aerosol turbidity over the ocean from geostationary satellite data. $J$. Appl. Meteor., 19, 633-644.

Reynolds, D. W., and T. H. Vonder Haar, 1977: A bi-spectral method 
for cloud parameter determination. Mon. Wea. Rev., 105, 446457.

Rossow, W. B., (Ed.), 1981: Clouds in climate: Modeling and satellite observational studies. Workshop Rep. NASA Goddard Institute for Space Studies, $222 \mathrm{pp}$.

and archival. J. Climate Appl. Meteor., 23, 1253-1257.

Saunders, R. W., 1985: Monthly mean cloudiness observed from METEOSAT-2. J. Climate Appl. Meteor., 24, 114-127.

Schiffer, R. A., and W. B. Rossow, 1983: The International Satellite Cloud Climatology Project (ISCCP): The first project of the World Climate Research Programme. Bull. Amer. Meteor. Soc., 64, 779-784.

Shenk, W. F., and V. V. Salomonson, 1972: A simulation study exploring the effects of sensor spatial resolution on estimates of cloud cover from satellites. J. Appl. Meteor., 12, 214-220.

Simmer, C., E. Raschke and E. Ruprecht, 1982: A method for determination of cloud properties from two-dimensional histograms. Ann. Meteor., 18, 130-132.
Wielicki, B. A., and J. A. Coakley, 1981: Cloud retrieval using infrared sounder data: Error analysis. J. Appl. Meteor., 20, 157-169.

World Climate Program, 1982a: Report of the planning meeting on International Satellite Cloud Climatology Project (ISCCP), Geneva, August 1982, WCP-28.

__, 1982b: The International Satellite Cloud Climatology Project (ISCCP) Preliminary Implementation plan (Rev. 1), WCP-35.

_ 1983a: Report of the First Session of the International Working Group on Data Management for the International Satellite Cloud Climatology Project (ISCCP), New York, December 1982, WCP42.

1983b: Report of the Second Session of the International Working Group on Data Management for the International Satellite Cloud Climatology Project (ISCCP), New York, April 1983, WCP-52.

1984: The International Satellite Cloud Climatology Project (ISCCP): Cloud analysis algorithm intercomparison. March 1984, WCP-73, pp. 74. 